\title{
Recovery and Long Term Functional Outcome in Subjects With Critical Illness Polyneuropathy and Myopathy: a Scoping Review
}

Domenico Intiso ( $\nabla$ d.intiso@operapadrepio.it )

Unit of Neurorehabilitation, IRCCS Casa Sollievo della Sofferenza, San Giovanni Rotondo (FG)

Marco Centra

Unit of Neurorehabilitation, IRCCS Casa Sollievo della Sofferenza, San Giovanni Rotondo (FG)

Michelangelo Bartolo

Unit of Neurorehabilitation, Habilita Care \& Research, Zingonia (Bergamo)

Maria Teresa Gatta

Unit of Neurorehabilitation, IRCCS Casa Sollievo della Sofferenza, San Giovanni Rotondo (FG)

Michele Gravina

Unit of Neurorehabilitation, IRCCS Casa Sollievo della Sofferenza, San Giovanni Rotondo (FG)

Filomena Di Rienzo

Unit of Neurorehabilitation, IRCCS Casa Sollievo della Sofferenza, San Giovanni Rotondo (FG)

Research Article

Keywords: critical illness polyneuropathy, ICU acquired weakness, functional outcome, rehabilitation

Posted Date: October 21st, 2021

DOI: https://doi.org/10.21203/rs.3.rs-952062/v1

License: (a) This work is licensed under a Creative Commons Attribution 4.0 International License. Read Full License 


\section{Abstract}

Background: Intensive care unit acquired weakness (ICUAW), embraces an array of disorders labeled "critical illness polyneuropathy" (CIP), "critical illness myopathy" (CIM) or "critical illness polyneuromyopathy" (CIPNM). Several studies have addressed the various characteristics of CIPNM, but the recovery is still unclear.

Objective: The present review investigated the recovery and the long-term functional outcome of subjects with CIPNM, whether the types of CIPNM have different outcomes and whether there is any supporting evidence.

Methods: Literature search was performed from MEDLINE/PubMed, CINAHL, EMBASE, PeDro, Web of Science and Scopus. Inclusion criteria were: i) sample size including five or more subjects; ii) subjects who suffered from CIPNM and/or CIP, CIM and CIP/CIM; iii) CIPNM ascertained by EMG. Follow-ups longer than one year were defined as long-term.

Results: Twenty-nine studies met the inclusion criteria. In total, 788 subjects with CIPNM were enrolled: 159 (20.1\%) died and 588 (74.6\%) were followed. Of all the included patients, 613 (77.7\%) had CIP, 82 (10.4\%) CIM and 56 (7.1\%) CIP/CIM. Overall, $70.3 \%$ of the subjects with CIPNM fully recovered. Seven (24.1\%) studies had a follow-up longer than 1 year (range 2-8) with 173 (21.9\%) subjects enrolled globally and 108 followed. Of these subjects, $88.8 \%$ gained full recovery. Most of the studies did not use proper functional scales and only 4 and 3 studies employed the Barthel scale and the FIM scale. Differentiation between the types of CIPNM was performed in 7 studies, but only 3 studies reported that subjects with CIM had a better prognosis and earlier recovery than subjects with CIP/CIM.

Conclusions: Subjects with CIPNM could achieve good recovery and could further improve at follow-up. The quality of the published studies due to short follow-ups and the paucity of defined outcome measures limit the evidence.

\section{Introduction}

A number of studies have been published about the muscle weakness that may affect intensive care unit (ICU) survivors. This disorder, which in the intensive care literature is better known as ICU acquired weakness (ICUAW), embraces a spectrum of clinical conditions. All of these conditions show variable levels of muscle strength impairment, from weakness to paralysis, which involve, bilaterally, the upper and lower limbs of ICU subjects. Although the clinical pictures are generally indistinguishable, ICUAW encompasses different pathological forms that damage the muscular and the peripheral nervous system. Different types of this pathology have been described and labeled according to the histological aspects and the electrophysiological findings and depending on the predominant structure involved. In particular, the definitions include: i) critical illness polyneuropathy (CIP), if the peripheral nervous system is affected; ii) critical illness myopathy (CIM), if the muscles are predominantly involved, and iii) critical illness polyneuropathy and myopathy (CIP/CIM), critical illness neuromyopathy [1], and polyneuromyopathy (CIPNM) [2-3], if the pathological process affects both muscles and nerves. Although ICU specialists prefer to use the term ICUAW, the definition "critical illness polyneuromyopathy" (CIPNM) is widely diffuse, and better suits the purposes of the present study. After the first description by Bolton et al. at the beginning of the '80s [4], a number of studies have been published that have contributed to making remarkable advances in the understanding of the complex aspects of CIPNM, such as the electrophysiological [5] and histological features [6] as well as the pathogenic mechanisms. The occurrence of this disorder has been variously detected with a range from 45 to $80 \%$ [7-9]. A systematic review reported a median prevalence of $43 \%$ (10). Furthermore, many risk factors have been suggested to favor the development of CIPNM, including sex (female), sepsis, ICU length of stay and multiple organ failure [11-12]. Several therapeutic approaches and strategies have been proposed and evaluated for the management of CIPNM subjects, but pharmacological treatments have failed to prevent the occurrence and were ineffective in treating the disorder [13-15]. However, recent reviews and meta-analyses have demonstrated that early mobilization is associated with a lower likelihood of developing this clinical condition [16-17]. Some reviews have addressed the various aspects of CIPNM, but they have not highlighted recovery, functional outcome and quality of life [18-20]. Despite the lack of treatments and the limitations of rehabilitative strategies, it was reported that 55-70\% of subjects reached a full recovery after ICU discharge [21-22], and that recovery depended on the type of CIPNM, as confirmed by the fact that CIM had an earlier and better functional outcome than CIP [22-23]. In 2005, an extensive literature review of the neuromuscular sequelae of ICU subjects with critical illness reported that $68.8 \%$ of them made a complete recovery and regained the ability to walk independently. However, such review was limited by an insufficient number of patients with a long follow-up, leaving unsettled the question of whether deficits following CIPNM were persistent [21]. Since then, no extensive study investigating the functional outcome in these subjects have been carried out, and the issue remains unsolved. The aim of the present review was to investigate recovery, in particular the longterm functional outcome of subjects with CIPNM, whether the types of CIPNM have different outcomes and whether there is any supporting evidence.

\section{Materials And Methods}

A search of the studies having tested the functional outcome in subjects with CIPNM was conducted using MEDLINE/PubMed, the Cochrane Central Register of Controlled Trials, CINAHL, EMBASE, PeDro, Web of Science and Scopus databases. The search was restricted to English language reports published between January 1984 and April 2021. The search terms varied slightly from database to database but included "intensive care unit acquired weakness", "ICUAW", "critical illness polyneuropathy", "CIP", "critical illness myopathy", "CIM", "critical polyneuropathy and myopathy", "CIPNM", “CIP and CIM"; “CIP/CIM", "acute tetraplegia”, "rehabilitation”, "functional outcome”, "recovery”, "physical therapy” and "mobilization”. Search limits included only adults. Conference abstracts/posters or articles that were not peer-reviewed were excluded. The literature search was conducted by 
three independent authors (MC, MB, FDR). Inclusion criteria were: i) sample size including five or more subjects; ii) subjects who suffered from CIPNM and/or the following types: CIP, CIM and CIP/CIM; iii) CIPNM ascertained by EMG; iv) studies with mixed samples that used the definition of ICUAW, but subjects with CIP, CIM or CIP/CIM were also considered; v) follow-up and outcome.

In order to avoid confounding results, studies were excluded if: i) they contained only the definition of the ICUAW, without any reference to the types of CIPNM or to CIP, CIM or CIP/CIM; ii) ICUAW was not ascertained by EMG; iii) reviews concerned ICUAW/CIPNM but the main aim of the study was not the outcome. Studies concerning subjects with CIPNM and COVID-19 were also excluded.

We defined as long-term follow-ups those follow-ups longer than 1 year. In this review, the pathological condition was counted as CIP if this acronym or definition was not specified in the studies analyzed. Due to the variability of the study designs, the functional measurements, the follow-ups, and the lack of data on the score of measurements, quantitative analysis was not possible. The research was conducted according to the Preferred Reporting Items for Systematic Reviews and the Meta-Analyses (PRISMA) diagram, depicting the selection of the articles searched for the study.

\section{Results}

The Prisma diagram of the studies' selection is shown in Figure 1. After studies were searched for and collected, 36 of them were considered eligible; of these, 29 [2, 6, 22, 23-48] were included according to inclusion and exclusion criteria (Table 1). Seven studies [49-55] were excluded even if they included subjects with CIPNM (Appendix 1). In particular, 4 of these 7 studies were excluded because they contained a duplicate of the data already included in the 29 studies, where they were analyzed in a greater sample [48-51]; 1 study with a large sample, due to the diagnosis of CIPNM being based predominantly on the clinical examination [52]; 2 studies including ICU patients having been discharged with the ICD-9/ICD-10 code for CIP and CIM (53-55) and did not use functional scale scores [52-53]. The included studies varied in aim, methodology design, sample size, case mix, inclusion/exclusion criteria, timing of the examination, follow-up and definition of recovery. Seventeen (58.6\%) studies had a prospective design. Twenty-four (82.7\%) studies concerned case series or small cohorts that had a mean sample size of $19.1 \pm 7.7$ patients and did not exceed the total number of 30 subjects. The other 5 studies [35, 41, 44- 45, 48] had samples greater than 30 patients (range 36-119). A total of 788 subjects with CIPNM were enrolled; of these 159 (20.1\%) died and 588 (74.6\%) were followed. All studies except 7 [6, 22-23, 35, 41, 44, 46] did not perform the differentiation between the types of CIPNM and considered the disorder as a unique entity, labelling it as CIP or, more generically, as polyneuropathy or neuromuscular disorder. In particular, 613 (77.7\%) patients had CIP, 82 (10.4\%) CIM and 56 (7.1\%) CIP/CIM. Diagnoses requiring ICU admission were widely and due to variable medical and surgical disorders. Two studies investigated the functional outcome in patients with CIPNM and coexistent brain lesions $[45,48]$. Twenty $(68.9 \%)$ studies were performed on subjects during their ICU or post-ICU stay, 1 in neurology and $8(27.5 \%)$ in rehabilitation or in neuro-rehabilitative settings. 
Table 1

Studies about functional outcome in CIPNM subjects. The pathological condition was counted as CIP if differentiation between types of CIPNM was not performed and if this acronym or definition was not specified in the studies analyzed.

\begin{tabular}{|c|c|c|c|c|c|c|c|}
\hline Authors & $\begin{array}{l}\text { Study design/ } \\
\text { Setting/aim }\end{array}$ & $\begin{array}{l}\text { N/followed/ } \\
\text { CIPNM type }\end{array}$ & Etiology & Follow-up & $\begin{array}{l}\text { Functional } \\
\text { measures/ } \\
\text { other }\end{array}$ & Other measures & Outcome \\
\hline $\begin{array}{l}\text { Zochodne } \\
\text { DW et al }{ }^{24} \\
(1987)\end{array}$ & $\begin{array}{l}\text { case series, } \\
\text { retrospective; } \\
\text { single center; } \\
\text { ICU; clinical and } \\
\text { electrophysiological } \\
\text { aspects }\end{array}$ & $\begin{array}{l}\mathrm{N}=19 ; \\
(9 \mathrm{M}, 10 \mathrm{~F} \\
\text { mean age } \\
64) ; \\
\mathrm{CIP}=19\end{array}$ & $\begin{array}{l}\text { cardiac or } \\
\text { pulmonary } \\
\text { diseases; } \\
5 \text { pts had } \\
\text { cerebral lesion } \\
\text { ( } 4 \text { infarction, } \\
1 \text { brain injury) }\end{array}$ & $\begin{array}{l}10 \text { mos-2 } \\
\text { yrs }\end{array}$ & none & $\begin{array}{l}\text { EMG, } \\
\text { histological } \\
\text { examination }\end{array}$ & $\begin{array}{l}8(88.8 \%) \text { pts } \\
\text { showed good } \\
\text { functional } \\
\text { recovery. Of these } \\
6 \text { pts had EMG } \\
\text { improvement } \\
\text { within } 3 \text { months. } \\
\text { At } 2 \text { yrs one } \\
\text { patients had } \\
\text { mildly weak } \\
\text { dorsiflexion of } \\
\text { right foot and one } \\
\text { had mild distal } \\
\text { limb weakness. } \\
11 \text { (58\%) pts died }\end{array}$ \\
\hline $\begin{array}{l}\text { Coronel B et } \\
\mathrm{al}^{25}(1990)\end{array}$ & $\begin{array}{l}\text { case series, } \\
\text { retrospective; } \\
2 \text { ICU center; } \\
\text { occurrence and } \\
\text { clinical features }\end{array}$ & $\begin{array}{l}\mathrm{N}=15 / 4 \\
(12 \mathrm{M}, 3 \mathrm{~F}, \\
\text { mean age } \\
47) ; \\
\mathrm{CIP}=15\end{array}$ & $\begin{array}{l}\text { cardiac or } \\
\text { pulmonary } \\
\text { disorders }\end{array}$ & $1-8 \mathrm{yrs}$ & none & $\begin{array}{l}\text { EMG, muscle } \\
\text { biopsy }\end{array}$ & $\begin{array}{l}1(25 \%) \text { pt had } \\
\text { complete recovery; } \\
3 \text { pts: } 2 \text { pts had } \\
\text { persisting } \\
\text { dysesthesia; } \\
1 \text { needing } \\
\text { assistance to sit } \\
\text { and walk; } \\
\text { Death: } 5 \text { pts }(33 \%)\end{array}$ \\
\hline $\begin{array}{l}\text { Op de Cul et } \\
\mathrm{al}^{2}(1991)\end{array}$ & $\begin{array}{l}\text { case series, } \\
\text { retrospective; } \\
\text { ICU; clinical and } \\
\text { electrophysiological } \\
\text { features }\end{array}$ & $\begin{array}{l}N=22^{*} ; \\
(17 \mathrm{M}, 5 \mathrm{~F}, \\
\text { mean age } \\
55) \\
\mathrm{CP}=22\end{array}$ & $\begin{array}{l}\text { Multiple trauma } \\
\text { with brain injury } \\
\text { (5 pts), } \\
\text { pulmonary and } \\
\text { infections }\end{array}$ & 2-10 mos & none & $\begin{array}{l}\text { EMG, muscle } \\
\text { biopsy ( } 7 \text { pts) }\end{array}$ & $\begin{array}{l}9(64.2 \%) \text { pts had } \\
\text { complete } \\
\text { functional } \\
\text { recovery; } 5 \\
(22.7 \%) \text { pts: } \\
\text { incomplete } \\
\text { recovery; } \\
8 \text { pts died. }\end{array}$ \\
\hline $\begin{array}{l}\text { Witt NJ et } \\
\mathrm{al}^{26}(1991)\end{array}$ & $\begin{array}{l}\text { case series, } \\
\text { prospective; } \\
\text { ICU; clinical and } \\
\text { electrophysiological } \\
\text { features }\end{array}$ & $\begin{array}{l}\mathrm{N}=30 ; \\
\mathrm{CIP}=30 \text {, of } \\
\text { these } 25 \text { had } \\
\text { clinical signs } \\
\text { of PN and } 15 \\
\text { pts ES signs }\end{array}$ & $\begin{array}{l}\text { multiple medical } \\
\text { and surgical } \\
\text { diseases; } 25 \% \\
\text { had head } \\
\text { trauma and } \\
\text { brain lesions }\end{array}$ & $\begin{array}{l}\text { mean } 72 \\
\text { days }(10- \\
190)\end{array}$ & none & EMG & $\begin{array}{l}20(66.6 \%) \text { pts } \\
\text { gained full } \\
\text { recovery; } \\
3(10 \%) \text { with } \\
\text { severe CIP } \\
\text { showed severe } \\
\text { disability and } \\
\text { ultimately died. }\end{array}$ \\
\hline
\end{tabular}

Legend: $A D L=$ activity daily living; $\mathrm{DRS}$ = disability rating scale; GOS = Glasgow outcome scale; dmCMAP= direct muscle stimulation; $E S=$ electrophysiological studies; FIM = Functional independence measure; ICF = International Classification of Functioning, Disability and Health; ICUAP = Intensive Care Unit acquired paresis; IPA = Impact on Participation and Autonomy questionnaire; LCF = Levels of Cognitive Functioning; LOS = length of stay; MRC = Medical Research Council scale; $m R S=$ modified Rankin Scale; ODSS = Overall Disability Sum score; ONLS = Overall Neuropathy Limitations Scale; neCMAP= nerve stimulation; RLOS = rehabilitation length of stay; RMI = Rivermead mobility index; $\mathrm{sABI}=$ severe acquired brain injury; SCI = spinal cord injury; SIP-68 = Sickness Impact Profile; SF 36= Short Form 36 questionnaire; TBI = traumatic brain injury; CRS-R = Coma Recovery Scale-Revised; GOS-E = Glasgow Outcome Scale-Expanded; FOIS = Functional Oral Intake Scale

${ }^{\circ}$ number of patients who had polyneuropathy to EMG; $£$ the sample included children and CIP was not defined; *including 12 patients described in a precedent paper; ${ }^{* *}$ other forms of myopathy or motor axonopathy could not be excluded; $\$$ description is reported in appendix 1 . 


\begin{tabular}{|c|c|c|c|c|c|c|c|}
\hline Authors & $\begin{array}{l}\text { Study design/ } \\
\text { Setting/aim }\end{array}$ & $\begin{array}{l}\text { N/followed/ } \\
\text { CIPNM type }\end{array}$ & Etiology & Follow-up & $\begin{array}{l}\text { Functional } \\
\text { measures/ } \\
\text { other }\end{array}$ & Other measures & Outcome \\
\hline $\begin{array}{l}\text { Rossiter A et } \\
\mathrm{al}^{27} \text { (1991) }\end{array}$ & $\begin{array}{l}\text { case reports, } \\
\text { retrospective; } \\
\text { single center; } \\
\text { ICU; clinical report } \\
\text { after pancuronium } \\
\text { discontinuation }\end{array}$ & $\begin{array}{l}\text { N= } 5 \text { pts; } \\
(4 \mathrm{M}, 1 \mathrm{~F}) \\
\text { CIPNM = } 5\end{array}$ & $\begin{array}{l}\text { medical } \\
\text { disorders }\end{array}$ & $5 \mathrm{mos}$ & none & $\begin{array}{l}\text { EMG; clinical } \\
\text { examination }\end{array}$ & $\begin{array}{l}\text { none had } \\
\text { complete } \\
\text { recovery: } \\
1 \text { pt severe } \\
\text { disability at } 3 \\
\text { months; } \\
1 \text { pt with } \\
\text { tetraparesis was } \\
\text { able to walk with } \\
\text { assistance at } 5 \\
\text { months; } \\
1 \text { pt with } \\
\text { tetraparesis was } \\
\text { unable to walk at } \\
1 \text { month; } \\
2 \text { pts died }\end{array}$ \\
\hline $\begin{array}{l}\text { Gooch JL et } \\
\mathrm{al}^{28}(1993)\end{array}$ & $\begin{array}{l}\text { case series, } \\
\text { retrospective; } \\
\text { paralysis after } \\
\text { neuromuscular } \\
\text { junction blockade }\end{array}$ & $\begin{array}{l}\mathrm{N}=12 \mathrm{f} ; \\
\text { age range } 3.5 \\
\text { mos-64 yrs; } \\
\mathrm{CIP}=12\end{array}$ & $\begin{array}{l}\text { medical } \\
\text { disorders }\end{array}$ & $3-6 \mathrm{mos}$ & none & $\begin{array}{l}\text { MRC; EMG, } \\
\text { muscle biopsy } \\
\text { (2 pts) }\end{array}$ & $\begin{array}{l}5(50 \%) \text { pts } \\
\text { recovered } \\
\text { completely; } \\
5 \text { pts incomplete } \\
\text { recovery; } \\
2 \text { pts died }\end{array}$ \\
\hline $\begin{array}{l}\text { Giostra E et } \\
\mathrm{al}^{29}(1994)\end{array}$ & $\begin{array}{l}\text { case series, } \\
\text { retrospective; } \\
\text { ICU; } \\
\text { paralysis after } \\
\text { neuromuscular } \\
\text { junction blockade }\end{array}$ & $\begin{array}{l}\mathrm{N}=9 ; \\
(6 \mathrm{M}, 3 \mathrm{~F} \\
\text { mean age } \\
65.6+10.3) ; \\
\mathrm{N}=\text { CIPNM }\end{array}$ & $\begin{array}{l}\text { medical and } \\
\text { pulmonary } \\
\text { disorders. }\end{array}$ & $4-52$ wks & none & $\begin{array}{l}\text { EMG, muscle } \\
\text { biopsies (7 pts) }\end{array}$ & $\begin{array}{l}5(55.5 \%) \text { pts had } \\
\text { complete recovery. } \\
\text { Even if recovery } \\
\text { was usual, } \\
\text { residual peroneal } \\
\text { palsy was } \\
\text { frequent }\end{array}$ \\
\hline $\begin{array}{l}\text { Leijten F et } \\
\mathrm{al}^{30}(1995)\end{array}$ & $\begin{array}{l}\text { prospective } \\
\text { cohort study; single } \\
\text { center ICU, post-ICU; } \\
\text { incidence and risk } \\
\text { factors }\end{array}$ & $\begin{array}{l}\mathrm{N}=29^{\circ} ; \\
\mathrm{CIP}=29 \\
(21 \mathrm{M} 8 \mathrm{~F} ; \\
\text { mean age } \\
59.7 \pm 13.9 \\
\text { years); } \\
\mathrm{N}=12 \\
\text { evaluated to } \\
\text { follow-up }\end{array}$ & $\begin{array}{l}\text { surgical and } \\
\text { medical } \\
\text { disorders; } \\
3 \text { pts cerebral } \\
\text { surgery, multiple } \\
\text { trauma }(n=9) \text {, } \\
\text { cardiac } \\
\text { resuscitation } \\
(n=5) \text {, } \\
\text { intracranial } \\
\text { hemorrhage } \\
(n=2)\end{array}$ & $1 \mathrm{yr}$ & $\begin{array}{l}\text { none; } \\
\text { endpoint was } \\
\text { strength } \\
\text { greater than } \\
\text { MRC grade } \\
4 / 5 \text { in all } \\
\text { muscles with } \\
\text { ability to } \\
\text { walk for more } \\
\text { than } 50 \text { m } \\
\text { without aid or } \\
\text { ataxia }\end{array}$ & $\begin{array}{l}\text { neurological } \\
\text { examination; } \\
\text { MRC; EMG; }\end{array}$ & $\begin{array}{l}7(58.3 \%) \text { patients } \\
\text { recovered ( } 4 \text { pts } \\
\text { within } 3 \text { days and } \\
4 \text { weeks, } 3 \text { pts } \\
\text { within } 4 \text { weeks } \\
\text { and } 1 \text { year; } \\
5(41.7 \%) \text { pts had } \\
\text { severe disability } \\
\text { after year; } \\
9(31 \%) \text { pts died }\end{array}$ \\
\hline
\end{tabular}

Legend: $A D L=$ activity daily living; $\mathrm{DRS}$ = disability rating scale; GOS = Glasgow outcome scale; dmCMAP= direct muscle stimulation; $E S=$ electrophysiological studies; FIM = Functional independence measure; ICF = International Classification of Functioning, Disability and Health; ICUAP = Intensive Care Unit acquired paresis; IPA = Impact on Participation and Autonomy questionnaire; LCF = Levels of Cognitive Functioning; LOS = length of stay; MRC = Medical Research Council scale; $m R S=$ modified Rankin Scale; ODSS = Overall Disability Sum score; ONLS = Overall Neuropathy Limitations Scale; neCMAP = nerve stimulation; RLOS = rehabilitation length of stay; RMI = Rivermead mobility index; $\mathrm{sABI}$ = severe acquired brain injury; SCI = spinal cord injury; SIP-68 = Sickness Impact Profile; SF 36= Short Form 36 questionnaire; TBI = traumatic brain injury; CRS-R = Coma Recovery Scale-Revised; GOS-E = Glasgow Outcome Scale-Expanded; FOIS = Functional Oral Intake Scale

${ }^{\circ}$ number of patients who had polyneuropathy to EMG; $£$ the sample included children and CIP was not defined; *including 12 patients described in a precedent paper; ${ }^{* *}$ other forms of myopathy or motor axonopathy could not be excluded; $\$$ description is reported in appendix 1. 


\begin{tabular}{|c|c|c|c|c|c|c|c|}
\hline Authors & $\begin{array}{l}\text { Study design/ } \\
\text { Setting/aim }\end{array}$ & $\begin{array}{l}\text { N/followed/ } \\
\text { CIPNM type }\end{array}$ & Etiology & Follow-up & $\begin{array}{l}\text { Functional } \\
\text { measures/ } \\
\text { other }\end{array}$ & Other measures & Outcome \\
\hline $\begin{array}{l}\text { Latronico N } \\
\text { et } \mathrm{al}^{31} \\
(1996)\end{array}$ & $\begin{array}{l}\text { case series, } \\
\text { prospective; } \\
\text { single center ICU; } \\
\text { incidence and risk } \\
\text { factors }\end{array}$ & $\begin{array}{l}N=24 ; \\
(19 \mathrm{M}, 5 \mathrm{~F}, \\
\text { mean age } \\
50.2 \pm 20.9 \\
\text { yrs); } \\
\text { CIPNM = } 24\end{array}$ & $\begin{array}{l}\text { All patients had } \\
\text { NCS lesions: } 13 \\
\text { head trauma; } \\
6 \text { subarachnoid } \\
\text { hemorrhage; } \\
3 \text { stroke; } 1 \\
\text { cerebral } \\
\text { hemorrhage }\end{array}$ & $8-18 \mathrm{mos}$ & none & $\begin{array}{l}\text { EMG/ENG; } \\
\text { nerve biopsy } \\
\text { (22 pts) }\end{array}$ & $\begin{array}{l}7 \text { survivors: } 6 \\
\text { ( } 85.7 \%) \text { had } \\
\text { recovered well or } \\
\text { had only } \\
\text { moderate } \\
\text { disability (able to } \\
\text { walk unassisted } \\
\text { with full muscle } \\
\text { strength); } \\
1 \text { was in } \\
\text { vegetative state; } \\
17(70.8 \%) \text { pts } \\
\text { died }\end{array}$ \\
\hline $\begin{array}{l}\text { Berek K et } \\
\text { al }^{32}(1996)\end{array}$ & $\begin{array}{l}\text { case series, } \\
\text { prospective; } \\
\text { ICU; } \\
\text { incidence, severity } \\
\text { and course of } \\
\text { polyneuropathies in } \\
\text { patients with sepsis } \\
\text { or systemic } \\
\text { inflammatory } \\
\text { response syndrome }\end{array}$ & $\begin{array}{l}\mathrm{N}=22 / 15 ; \\
(17 \mathrm{M}, 5 \mathrm{~F}, \\
\text { mean age } \\
51.2 \mathrm{yrs}) ; \\
\text { CIPNM = } 18\end{array}$ & $\begin{array}{l}\text { medical and } \\
\text { surgical } \\
\text { disorders }\end{array}$ & $2-3$ mos & $\begin{array}{l}\text { functional } \\
\text { disability } \\
\text { score }^{\$}\end{array}$ & EMG & $\begin{array}{l}9(50 \%) \text { pts had } \\
\text { complete } \\
\text { functional } \\
\text { recovery; } \\
6 \text { pts had } \\
\text { incomplete } \\
\text { functional } \\
\text { recovery. Of these, } \\
4 \text { pts had mild } \\
\text { weakness and } 2 \\
\text { pts had moderate } \\
\text { weakness. } \\
\text { Good tendency for } \\
\text { recovery in all } \\
\text { surviving patients, } \\
\text { electrophysiologic } \\
\text { findings were still } \\
\text { pathologic in } 11 \\
\text { patients at the } \\
\text { follow-up; } \\
7 \text { (50\%) pts died }\end{array}$ \\
\hline $\begin{array}{l}\text { Hund EF et } \\
\mathrm{al}^{33}(1996)\end{array}$ & $\begin{array}{l} \\
\text { single center } \\
\text { ICU; }\end{array}$ & $\begin{array}{l}N=7 \\
(3 \mathrm{M}, 4 \mathrm{~F} ; \\
\text { mean age } \\
47.7 \pm 16.8\end{array}$ & $\begin{array}{l}\text { medical } \\
\text { disorders; } \\
3 \text { pts with } \\
\text { cerebral lesions }\end{array}$ & $\begin{array}{l}3 \text { mos - } 3.5 \\
\text { yrs }\end{array}$ & none & $\begin{array}{l}\text { EMG; muscle } \\
\text { and nerve } \\
\text { biopsy ( } 3 \text { pts) }\end{array}$ & $\begin{array}{l}2 \text { ( } 40 \%) \text { pt gained } \\
\text { complete recovery; } \\
3 \text { pts showed } \\
\text { disability due to } \\
\text { CNS lesions; } \\
2 \text { pts died }\end{array}$ \\
\hline
\end{tabular}

Legend: $A D L=$ activity daily living; $\mathrm{DRS}$ = disability rating scale; GOS = Glasgow outcome scale; dmCMAP= direct muscle stimulation; $E S=$ electrophysiological studies; FIM = Functional independence measure; ICF = International Classification of Functioning, Disability and Health; ICUAP = Intensive Care Unit acquired paresis; IPA = Impact on Participation and Autonomy questionnaire; LCF = Levels of Cognitive Functioning; LOS = length of stay; MRC = Medical Research Council scale; $m R S=$ modified Rankin Scale; ODSS = Overall Disability Sum score; ONLS = Overall Neuropathy Limitations Scale; neCMAP = nerve stimulation; RLOS = rehabilitation length of stay; RMI = Rivermead mobility index; $\mathrm{sABI}$ = severe acquired brain injury; SCI = spinal cord injury; SIP-68 = Sickness Impact Profile; SF 36= Short Form 36 questionnaire; TBI = traumatic brain injury; CRS-R = Coma Recovery Scale-Revised; GOS-E = Glasgow Outcome Scale-Expanded; FOIS = Functional Oral Intake Scale

${ }^{\circ}$ number of patients who had polyneuropathy to EMG; $£$ the sample included children and CIP was not defined; *including 12 patients described in a precedent paper; ${ }^{* *}$ other forms of myopathy or motor axonopathy could not be excluded; $\$$ description is reported in appendix 1. 


\begin{tabular}{|c|c|c|c|c|c|c|c|}
\hline Authors & $\begin{array}{l}\text { Study design/ } \\
\text { Setting/aim }\end{array}$ & $\begin{array}{l}\text { N/followed/ } \\
\text { CIPNM type }\end{array}$ & Etiology & Follow-up & $\begin{array}{l}\text { Functional } \\
\text { measures/ } \\
\text { other }\end{array}$ & Other measures & Outcome \\
\hline $\begin{array}{l}\text { Campellone } \\
\text { JV et al }{ }^{34} \\
(1998)\end{array}$ & $\begin{array}{l}\text { case series, } \\
\text { prospective; single } \\
\text { center ICU; } \\
\text { frequency of } \\
\text { myopathy as a } \\
\text { cause of } \\
\text { generalized } \\
\text { weakness and } \\
\text { potential risk } \\
\text { factors after } \\
\text { liver transplant }\end{array}$ & $\begin{array}{l}\mathrm{N}=7 / 6 ; \\
(6 \mathrm{M}, 1 \mathrm{~F} ; \\
\text { mean age } \\
57.7 \pm 9.3) \\
\mathrm{CIM}=7\end{array}$ & liver transplant & $\begin{array}{l}11-41 \text { days } \\
(5 \mathrm{pts}) \text { and } \\
67 \text { days ( } 1 \\
\text { pt) }\end{array}$ & none & $\begin{array}{l}\text { EMG; muscle } \\
\text { biopsies (5 pts) }\end{array}$ & $\begin{array}{l}3(50 \%) \text { pts } \\
\text { regained strength } \\
\text { slowly and were } \\
\text { able to ambulate } \\
\text { within } 4 \text { to } 12 \\
\text { weeks; } \\
1 \text { pt required a } \\
\text { walker; } \\
2 \text { pts died }\end{array}$ \\
\hline $\begin{array}{l}\text { Lacomis D. } \\
\text { et al }{ }^{35} \\
(1997)\end{array}$ & $\begin{array}{l}\text { cohort, } \\
\text { retrospective; single } \\
\text { center ICU; } \\
\text { causes of ICU } \\
\text { weakness }\end{array}$ & $\begin{array}{l}\mathrm{N}=92 ; \\
\mathrm{N}=49 \\
\mathrm{CIM}=37 \\
\mathrm{CIP}=12\end{array}$ & $\begin{array}{l}\text { surgical, } \\
\text { medical and } \\
\text { pulmonary } \\
\text { disorders }\end{array}$ & $\begin{array}{l}12-60 \\
\text { mos }\end{array}$ & none & $\begin{array}{l}\text { EMG; muscle } \\
\text { biopsies ( } 22 \\
\text { pts). }\end{array}$ & $\begin{array}{l}25(75.7 \%) \text { pts } \\
\text { had complete } \\
\text { functional } \\
\text { recovery: } 17 \text { pts } \\
\text { were ambulatory } \\
\text { within } 4 \text { months } \\
\text { and } 8 \text { pts within } 4- \\
12 \text { months; } \\
7 \text { pts showed } \\
\text { incomplete } \\
\text { functional } \\
\text { recovery: } 4 \\
\text { remained non } \\
\text { ambulatory and } 3 \\
\text { remained } \\
\text { dependent on the } \\
\text { ventilator; } \\
16 \text { pts died }\end{array}$ \\
\hline $\begin{array}{l}\text { de Sèze M. } \\
\text { et al }{ }^{36} \\
(2000)\end{array}$ & $\begin{array}{l}\text { cohort, } \\
\text { retrospective; } \\
\text { single center; } \\
\text { rehabilitation; the } \\
\text { features and } \\
\text { outcome patients } \\
\text { who had severe } \\
\text { forms of CIP }\end{array}$ & $\begin{array}{l}\mathrm{N}=19 \text {, only } \\
\text { CIP (14 M } 5 \\
\mathrm{~F} ; \text { mean age } \\
55,9 \text { yrs) }\end{array}$ & $\begin{array}{l}\text { medical } \\
\text { disorders }\end{array}$ & $2 \mathrm{yrs}$ & none & $\begin{array}{l}\text { MRC; } \\
\text { sensory } \\
\text { findings }\end{array}$ & $\begin{array}{l}11(64.7 \%) \\
\text { patients recovered } \\
\text { completely; } \\
4(23.5 \%) \text { patients } \\
\text { remained } \\
\text { quadriplegic; } 2 \\
\text { patients remained } \\
\text { quadriparetic; }\end{array}$ \\
\hline
\end{tabular}

Legend: $A D L=$ activity daily living; $D R S$ = disability rating scale; $G O S=$ Glasgow outcome scale; dmCMAP= direct muscle stimulation; $E S=$ electrophysiological studies; FIM = Functional independence measure; ICF = International Classification of Functioning, Disability and Health; ICUAP = Intensive Care Unit acquired paresis; IPA = Impact on Participation and Autonomy questionnaire; LCF = Levels of Cognitive Functioning; LOS = length of stay; $M R C=$ Medical Research Council scale; $m R S$ = modified Rankin Scale; ODSS = Overall Disability Sum score; ONLS = Overall Neuropathy Limitations Scale; neCMAP = nerve stimulation; RLOS = rehabilitation length of stay; RMI = Rivermead mobility index; $\mathrm{SABI}=$ severe acquired brain injury; SCI = spinal cord injury; SIP-68 = Sickness Impact Profile; SF 36= Short Form 36 questionnaire; TBI = traumatic brain injury; CRS-R = Coma Recovery Scale-Revised; GOS-E = Glasgow Outcome Scale-Expanded; FOIS = Functional Oral Intake Scale

${ }^{\circ}$ number of patients who had polyneuropathy to EMG; $£$ the sample included children and CIP was not defined; *including 12 patients described in a precedent paper; ${ }^{* *}$ other forms of myopathy or motor axonopathy could not be excluded; $\$$ description is reported in appendix 1 . 


\begin{tabular}{|c|c|c|c|c|c|c|c|}
\hline Authors & $\begin{array}{l}\text { Study design/ } \\
\text { Setting/aim }\end{array}$ & $\begin{array}{l}\text { N/followed/ } \\
\text { CIPNM type }\end{array}$ & Etiology & Follow-up & $\begin{array}{l}\text { Functional } \\
\text { measures/ } \\
\text { other }\end{array}$ & Other measures & Outcome \\
\hline $\begin{array}{l}\text { Zifko UA et } \\
\mathrm{al}^{37}(2000)\end{array}$ & $\begin{array}{l}\text { cohort, } \\
\text { retrospective; } \\
\text { ICU and } \\
\text { rehabilitation; } \\
\text { clinical outcome } \\
\text { and } \\
\text { electrophysiological } \\
\text { findings }\end{array}$ & $\begin{array}{l}\mathrm{N}=26 ; \\
\mathrm{CIP}=13,(9 \mathrm{M}, \\
4 \mathrm{~F}, \text { age } \\
\text { between } 22- \\
83 \text { yrs); } \\
\mathrm{N}=7 \text { refused } \\
\text { to participate; }\end{array}$ & $\begin{array}{l}\text { medical } \\
\text { disorders; } \\
1 \text { pt with stroke }\end{array}$ & $\begin{array}{l}13-24 \text { mos } \\
\text { (mean } 17 \\
\text { mos) }\end{array}$ & none & $\begin{array}{l}\text { EMG/ENG; } \\
\text { MRC; clinical } \\
\text { examination }\end{array}$ & $\begin{array}{l}\text { only } 2(15.3 \%) \text { pts } \\
\text { had full recovery; } \\
11 \text { of } 13 \text { patients } \\
\text { with CIP had } \\
\text { clinical } \\
\text { manifestations, at } \\
\text { follow-up (13-24 } \\
\text { months after } \\
\text { diagnosis); } \\
6 \text { pts died }\end{array}$ \\
\hline $\begin{array}{l}16 \mathrm{De} \\
\text { Jonghe B. et } \\
\mathrm{al}^{38}(2002)\end{array}$ & $\begin{array}{l}\text { cohort, prospective; } \\
\text { multicenter } \\
\text { ICU and post-ICU; } \\
\text { clinical incidence, } \\
\text { risk factors, and } \\
\text { outcomes of ICU } \\
\text { acquired paresis } \\
\text { (ICUAP) during ICU } \\
\text { stay }\end{array}$ & $\begin{array}{l}\mathrm{N}=95 ; \\
\text { CIP (ICUAP)= } \\
24 ; \\
(12 \mathrm{M}, 12 \mathrm{~F} ; \\
\text { mean age } \\
67,6 \text { yrs) }\end{array}$ & $\begin{array}{l}\text { surgical and } \\
\text { medical } \\
\text { disorders; } \\
\text { patients were } \\
\text { excluded if they } \\
\text { had disease of } \\
\text { the peripheral } \\
\text { nervous system, } \\
\text { or brainstem } \\
\text { lesions }\end{array}$ & $9 \mathrm{mos}$ & none & $\begin{array}{l}\text { MRC; EMG; } \\
\text { muscle biopsy } \\
\text { (10 pts) }\end{array}$ & $\begin{array}{l}15(88.6 \%) \\
\text { patients had } \\
\text { recovered an MRC } \\
\text { score of } 48 \text { or } \\
\text { higher at follow- } \\
\text { up; } \\
1 \text { pt lost to follow- } \\
\text { up; } \\
7 \text { pts died }\end{array}$ \\
\hline $\begin{array}{l}\text { Fletcher S.N. } \\
\text { et al }{ }^{39} \\
(2003)\end{array}$ & $\begin{array}{l}\text { cohort, prospective } \\
\text { study; multicenter } \\
\text { post-ICU; } \\
\text { prevalence, clinical } \\
\text { characteristics } \\
\text { and } \\
\text { electrophysiological } \\
\text { features }\end{array}$ & $\begin{array}{l}\mathrm{N}=22 ; \\
\mathrm{CIP}=22 ; \\
\text { (mean age } 62 \\
\text { yrs, range 45- } \\
78 \text { ); }\end{array}$ & $\begin{array}{l}\text { surgical and } \\
\text { medical } \\
\text { disorders }\end{array}$ & $\begin{array}{l}3.5 \mathrm{yrs} \\
\text { (range, 12- } \\
57 \text { mos) }\end{array}$ & Barthel Index & $\begin{array}{l}\text { neurologic } \\
\text { examination; } \\
\text { EMG }\end{array}$ & $\begin{array}{l}19(86.3 \%) \text { pts } \\
\text { had full recovery } \\
\text { (BI score } 95-100) ; \\
2 \text { pts incomplete } \\
\text { recovery (BI score } \\
85) ; \\
1 \text { pts severe } \\
\text { disabled. 95\% } \\
\text { patients had } \\
\text { electromyographic } \\
\text { evidence of } \\
\text { chronic partial } \\
\text { denervation, } \\
\text { indicative of a } \\
\text { preceding axonal } \\
\text { neuropathy }\end{array}$ \\
\hline $\begin{array}{l}\text { Kerbaul et } \\
\text { al }^{6}(2004)\end{array}$ & $\begin{array}{l}\text { cohort, } \\
\text { prospective; } \\
\text { single center post- } \\
\text { ICU; to describe } \\
\text { patterns of } \\
\text { neuromuscular } \\
\text { weakness by EMG } \\
\text { and biopsy; } \\
\text { functional outcome }\end{array}$ & $\begin{array}{l}\mathrm{N}=15 \mathrm{pts} ; \\
(9 \mathrm{M}, 6 \mathrm{~F} ; \\
\text { median age } \\
53 \text { yrs, range } \\
33-82) ; \\
\mathrm{CIP}=6 \\
\mathrm{CIM}=6 ; \\
\mathrm{CIP} / \mathrm{CIM}=3\end{array}$ & heart-surgery & 12 mos & $\begin{array}{l}\text { none, } \\
\text { the endpoints } \\
\text { were death or } \\
\text { time to } \\
\text { ambulation } \\
\text { without } \\
\text { assistance; }\end{array}$ & $\begin{array}{l}\text { EMG; } \\
\text { muscular/nerve } \\
\text { biopsy (all pts) }\end{array}$ & $\begin{array}{l}6(75 \%) \text { had good } \\
\text { recovery; } 2 \\
\text { subjects of the } 8 \\
\text { survivors were not } \\
\text { ambulatory; } \\
7(46 \%) \text { pts died }\end{array}$ \\
\hline
\end{tabular}

Legend: $A D L=$ activity daily living; $\mathrm{DRS}$ = disability rating scale; GOS = Glasgow outcome scale; dmCMAP= direct muscle stimulation; $E S=$ electrophysiological studies; FIM = Functional independence measure; ICF = International Classification of Functioning, Disability and Health; ICUAP = Intensive Care Unit acquired paresis; IPA = Impact on Participation and Autonomy questionnaire; LCF = Levels of Cognitive Functioning; LOS = length of stay; MRC = Medical Research Council scale; $m R S=$ modified Rankin Scale; ODSS = Overall Disability Sum score; ONLS = Overall Neuropathy Limitations Scale; neCMAP = nerve stimulation; RLOS = rehabilitation length of stay; RMI = Rivermead mobility index; $\mathrm{sABI}$ = severe acquired brain injury; SCI = spinal cord injury; SIP-68 = Sickness Impact Profile; SF 36= Short Form 36 questionnaire; TBI = traumatic brain injury; CRS-R = Coma Recovery Scale-Revised; GOS-E = Glasgow Outcome Scale-Expanded; FOIS = Functional Oral Intake Scale

${ }^{\circ}$ number of patients who had polyneuropathy to EMG; $£$ the sample included children and CIP was not defined; *including 12 patients described in a precedent paper; ${ }^{* *}$ other forms of myopathy or motor axonopathy could not be excluded; $\$$ description is reported in appendix 1 . 


\begin{tabular}{|c|c|c|c|c|c|c|c|}
\hline Authors & $\begin{array}{l}\text { Study design/ } \\
\text { Setting/aim }\end{array}$ & $\begin{array}{l}\text { N/followed/ } \\
\text { CIPNM type }\end{array}$ & Etiology & Follow-up & $\begin{array}{l}\text { Functional } \\
\text { measures/ } \\
\text { other }\end{array}$ & Other measures & Outcome \\
\hline $\begin{array}{l}\text { Van der } \\
\text { schaaf } M \text { et } \\
\text { al }^{40}(2004)\end{array}$ & $\begin{array}{l}\text { prospective } \\
\text { observational } \\
\text { cohort study + } \\
\text { cross-sectional } \\
\text { study; single center } \\
\text { ICU, post-ICU; to } \\
\text { evaluate the } \\
\text { functional outcome } \\
\text { of ICU patients }\end{array}$ & $\begin{array}{l}\mathrm{N}=16 ; \\
(12 \mathrm{M}, 4 \mathrm{~F} ; \\
\text { mean age } 67 \\
\text { years); } \\
\mathrm{CIP}=16\end{array}$ & $\begin{array}{l}\text { medical and } \\
\text { surgical } \\
\text { disorders; } \\
\text { patients with } \\
\text { neurological } \\
\text { disorders due } \\
\text { CNS injury were } \\
\text { excluded }\end{array}$ & $\begin{array}{l}6 \mathrm{mos} \text { and } \\
1 \mathrm{yr}\end{array}$ & $\begin{array}{l}\text { Barthel Index; } \\
\text { Jebsen hand } \\
\text { function test; } \\
\text { rivermead } \\
\text { mobility } \\
\text { index; } \\
\text { timed UP \& } \\
\text { GO walking } \\
\text { test }\end{array}$ & $\begin{array}{l}\text { MRC; SIP-68; } \\
\text { SF-36; } \\
\text { IPA } \\
\text { questionnaire }\end{array}$ & $\begin{array}{l}\text { At } 6 \text { mos, } 8 \text { pts } \\
\text { were evaluated } \\
\text { and all showed } \\
\text { moderate } \\
\text { disability (activity } \\
\text { and participation); } \\
\text { at } 1 \text { yr, } 5 \text { (31.2\%) } \\
\text { pts were } \\
\text { evaluated: } \\
\text { improvement in } \\
\text { functional abilities } \\
\text { with wide } \\
\text { variation in } \\
\text { functional } \\
\text { outcome among } \\
\text { the patients; } \\
\text { functional } \\
\text { impairment was } \\
\text { still dominant in } \\
\text { four out of } 5 \\
\text { surviving pts. } \\
\text { Outdoor mobility } \\
\text { was reduced; } \\
9 \text { (56.2\%) patients } \\
\text { died }\end{array}$ \\
\hline $\begin{array}{l}\text { Guarneri B et } \\
\mathrm{al}^{22}(2008)\end{array}$ & $\begin{array}{l}\text { prospective cohort; } \\
\text { multicenter post- } \\
\text { ICU; to evaluate the } \\
\text { long-term } \\
\text { follow-up }\end{array}$ & $\begin{array}{l}\mathrm{N}=92 ; \\
\mathrm{CIP}=15 \\
(12 \mathrm{M} 3 \mathrm{~F} ; \\
\text { mean age } \\
44.7 \pm 14.9 \\
\mathrm{yrs}) ; \\
\mathrm{CIP}=4 \\
\mathrm{CIM}=6 \\
\mathrm{CIP} / \mathrm{CIM}=3 \\
2= \\
\text { undetermined }\end{array}$ & $\begin{array}{l}\text { surgical and } \\
\text { medical } \\
\text { disorders; } \\
\text { (intracerebral } \\
\text { haemorrhage, } \\
\text { metabolic } \\
\text { encephalopathy, } \\
\text { post-anoxic } \\
\text { encephalopathy: } \\
1 \text { patient each); } \\
5 \text { multiple } \\
\text { trauma patients; } \\
3 \text { head trauma }\end{array}$ & $1 \mathrm{yr}$ & $\begin{array}{l}\text { global motor } \\
\text { performance }\end{array}$ & $\begin{array}{l}\text { MRC; EMG; } \\
\text { neurological } \\
\text { examination }\end{array}$ & $\begin{array}{l}8(61.5 \%) \text { patients } \\
\text { recovered; } 2 \\
(13.3 \%) \text { patients } \\
\text { had persisting } \\
\text { muscle weakness; } \\
1 \text { patient } \\
\text { remained } \\
\text { tetraparetic; } 1 \\
\text { patient remained } \\
\text { tetraplegic; } \\
1 \text { patient lost to } \\
\text { follow-up; } \\
2 \text { patients died; }\end{array}$ \\
\hline $\begin{array}{l}\text { Intiso D et } \\
\text { al }^{41}(2011)\end{array}$ & $\begin{array}{l}\text { cohort prospective; } \\
\text { single center neuro- } \\
\text { rehabilitation; } \\
\text { to evaluate the } \\
\text { long-term } \\
\text { functional outcome } \\
\text { and health status }\end{array}$ & $\begin{array}{l}\mathrm{N}=42(23 \mathrm{M}, \\
19 \mathrm{~F}, \text { mean } \\
\text { age } 58.4 \pm \\
13.9) ; \\
\mathrm{CIP}=30 \mathrm{CIM}= \\
6 \mathrm{CIP} / \mathrm{CIM}=6\end{array}$ & $\begin{array}{l}19 \text { pts had CNS } \\
\text { damage }\end{array}$ & $\begin{array}{l}5 \text { yrs; } \\
\text { mean } \\
31.7 \pm 15.8 \\
\text { months }\end{array}$ & $\begin{array}{l}\text { Barthel and } \\
\text { modified } \\
\text { Rankin } \\
\text { Scales } \\
\text { (mRS); }\end{array}$ & $\begin{array}{l}\text { SF-36 } \\
\text { questionnaire }\end{array}$ & $\begin{array}{l}31(73.8 \%) \text { pts ( } 24 \\
\text { pts with just } \\
\text { CIPNM and } 7 \text { pts } \\
\text { with CIPNM and } \\
\text { CNS involvement) } \\
\text { gained good } \\
\text { recovery: mean } \\
\text { Barthel of } \\
86.7 \pm 15.9 \text { (P< } \\
0.001) \text {, and the } \\
\text { median mRS of } 1 \\
\text { (IQR: } 0-3 \text { ), } \\
\text { respectively, at } \\
\text { follow-up (mean } \\
31.7 \pm 15.8 \\
\text { months) }\end{array}$ \\
\hline
\end{tabular}

Legend: $A D L$ = activity daily living; $D R S$ = disability rating scale; GOS = Glasgow outcome scale; dmCMAP= direct muscle stimulation; ES= electrophysiological studies; FIM = Functional independence measure; ICF = International Classification of Functioning, Disability and Health; ICUAP = Intensive Care Unit acquired paresis; IPA = Impact on Participation and Autonomy questionnaire; LCF = Levels of Cognitive Functioning; LOS = length of stay; MRC = Medical Research Council scale; $m R S=$ modified Rankin Scale; ODSS = Overall Disability Sum score; ONLS = Overall Neuropathy Limitations Scale; neCMAP = nerve stimulation; RLOS = rehabilitation length of stay; RMI = Rivermead mobility index; $\mathrm{sABI}$ = severe acquired brain injury; SCI = spinal cord injury; SIP-68 = Sickness Impact Profile; SF 36= Short Form 36 questionnaire; TBI = traumatic brain injury; CRS-R = Coma Recovery Scale-Revised; GOS-E = Glasgow Outcome Scale-Expanded; FOIS = Functional Oral Intake Scale

${ }^{\circ}$ number of patients who had polyneuropathy to EMG; $£$ the sample included children and CIP was not defined; *including 12 patients described in a precedent paper; ${ }^{* *}$ other forms of myopathy or motor axonopathy could not be excluded; $\$$ description is reported in appendix 1 . 


\begin{tabular}{|c|c|c|c|c|c|c|c|}
\hline Authors & $\begin{array}{l}\text { Study design/ } \\
\text { Setting/aim }\end{array}$ & $\begin{array}{l}\text { N/followed/ } \\
\text { CIPNM type }\end{array}$ & Etiology & Follow-up & $\begin{array}{l}\text { Functional } \\
\text { measures/ } \\
\text { other }\end{array}$ & Other measures & Outcome \\
\hline $\begin{array}{l}\text { Novak P et } \\
\text { al }^{42}(2011)\end{array}$ & $\begin{array}{l}\text { cohort, } \\
\text { prospective; } \\
\text { single center } \\
\text { rehabilitation; } \\
\text { outcome to ICF }\end{array}$ & $\begin{array}{l}\mathrm{N}=27 ; \\
(16 \mathrm{~F}, 11 \mathrm{M} ; \\
\text { mean age } \\
59.4 \pm 15.9) ; \\
\mathrm{CIP}=27\end{array}$ & not reported & $\begin{array}{l}\text { from } \\
\text { admission } \\
\text { to } \\
\text { discharge } \\
\text { (9-102 } \\
\text { days) }\end{array}$ & $\begin{array}{l}\text { FIM; 6-min } \\
\text { (expressed in } \\
\text { meters) and } \\
\text { 10-meters } \\
\text { walking test } \\
\text { (expressed in } \\
\text { speed } \\
\text { velocity); ICF } \\
\text { check list }\end{array}$ & $\begin{array}{l}\text { sum of } \\
\text { muscles } \\
\text { strength; }\end{array}$ & $\begin{array}{l}\text { Significant } \\
\text { functional } \\
\text { improvement; } \\
\text { mean FIM score } \\
78.7 \pm 24.12 \text { and } \\
103.3 \pm 20.5 \text { at } \\
\text { admission and } \\
\text { discharge, } \\
\text { respectively } \\
\text { (p<0.001); } 6 \text {-min } \\
\text { walking test (m): } \\
77.3 \pm 115.3 \text { and } \\
191.5 \pm 178.2 \text { at } \\
\text { admission and } \\
\text { discharge, } \\
\text { respectively } \\
\text { (p<0.001). } \\
\text { Considering ICF, } \\
26(96.2 \%) \text { pts } \\
\text { improved } \\
\text { activities and } \\
\text { participation }\end{array}$ \\
\hline $\begin{array}{l}\text { Semmler A. } \\
\text { et al }{ }^{43} \\
(2013)\end{array}$ & $\begin{array}{l}\text { cohort, } \\
\text { retrospective } \\
\text { observational; } \\
\text { single center post- } \\
\text { ICU; long-term } \\
\text { outcome }\end{array}$ & $\begin{array}{l}\mathrm{N}=51 ; \\
(26 \mathrm{M}, 24 \mathrm{~F} ; \\
\text { median age } \\
57 \text { yrs, range } \\
19-75) ; \\
\mathrm{CIP}=21 \\
\text { no CIM or } \\
\text { CIP/CIM }\end{array}$ & $\begin{array}{l}\text { Subjects with } \\
\text { CNS lesion were } \\
\text { excluded }\end{array}$ & $\begin{array}{l}6-24 \text { mos, } \\
\text { median } 11 \\
\text { mos }\end{array}$ & $\begin{array}{l}\text { ODSS\$; } \\
\text { median } \\
\text { ODSS scores } \\
1 \text { (range 0- } \\
8 \text { ); }\end{array}$ & $\begin{array}{l}\text { MRC; median } \\
\text { MRC sum } \\
\text { scores 56 } \\
\text { (range 47-60); } \\
\text { EMG/ENG; } \\
\text { neurological } \\
\text { examination }\end{array}$ & $\begin{array}{l}\text { Good recovery; pts } \\
\text { with diagnosis of } \\
\text { CIP showed a } \\
\text { higher ODSS } \\
\text { scores } 1 \text { and } \\
\text { lower MRC sum } \\
\text { scores 56; } \\
\text { The } \\
\text { neuromuscular } \\
\text { long-term } \\
\text { consequences of } \\
\text { critical illness } \\
\text { were not severe, } \\
\text { suggesting a } \\
\text { favorable } \\
\text { prognosis of ICU- } \\
\text { acquired muscular } \\
\text { weakness }\end{array}$ \\
\hline $\begin{array}{l}\text { Koch S et } \\
\mathrm{al}^{23}(2014)\end{array}$ & $\begin{array}{l}\text { Prospective cohort; } \\
\text { post-ICU; prediction } \\
\text { of long-term } \\
\text { outcome in CIP and } \\
\text { CIM }\end{array}$ & $\begin{array}{l}\mathrm{N}=26 ; \\
(20 \mathrm{M}, 6 \mathrm{~F} ; \\
\text { mean age } 46 \\
\text { yrs); } \\
\mathrm{CIM}=8, \\
\mathrm{CIP} / \mathrm{CIM}=11 \text {, } \\
\text { Control = } 7\end{array}$ & $\begin{array}{l}\text { multiple trauma } \\
(n=12)\end{array}$ & $\begin{array}{l}1 \mathrm{yr} ; \\
\text { (mean } \\
411 \pm 121 \\
\text { days) }\end{array}$ & $\begin{array}{l}\text { functional } \\
\text { health } \\
\text { status } \$\end{array}$ & $\begin{array}{l}\text { MRC; EMG; } \\
\text { dmMCAP, } \\
\text { neCMAP; } \\
\text { neurological } \\
\text { examination }\end{array}$ & $\begin{array}{l}4(50 \%) \text { of the CIM } \\
\text { patients reached } \\
\text { normal physical } \\
\text { capacity. In } \\
\text { contrast, only } 3 \\
(27 \%) \text { of CIM/CIP } \\
\text { patients did so at } \\
1 \text { year. Four ( } 36 \%) \\
\text { of CIM/CIP pts } \\
\text { still needed } \\
\text { assistance to } \\
\text { perform daily life } \\
\text { activities: } 2 \text { pts } \\
\text { were able to walk } \\
\text { only within their } \\
\text { homes and } 2 \text { were } \\
\text { only able to stand } \\
\text { with help or not at } \\
\text { all. }\end{array}$ \\
\hline
\end{tabular}

Legend: $A D L=$ activity daily living; $\mathrm{DRS}$ = disability rating scale; GOS = Glasgow outcome scale; dmCMAP= direct muscle stimulation; $E S=$ electrophysiological studies; FIM = Functional independence measure; ICF = International Classification of Functioning, Disability and Health; ICUAP = Intensive Care Unit acquired paresis; IPA = Impact on Participation and Autonomy questionnaire; LCF = Levels of Cognitive Functioning; LOS = length of stay; MRC = Medical Research Council scale; $m R S=$ modified Rankin Scale; ODSS = Overall Disability Sum score; ONLS = Overall Neuropathy Limitations Scale; neCMAP = nerve stimulation; RLOS = rehabilitation length of stay; RMI = Rivermead mobility index; $\mathrm{sABI}$ = severe acquired brain injury; SCI = spinal cord injury; SIP-68 = Sickness Impact Profile; SF 36= Short Form 36 questionnaire; TBI = traumatic brain injury; CRS-R = Coma Recovery Scale-Revised; GOS-E = Glasgow Outcome Scale-Expanded; FOIS = Functional Oral Intake Scale

${ }^{\circ}$ number of patients who had polyneuropathy to EMG; $£$ the sample included children and CIP was not defined; *including 12 patients described in a precedent paper; ${ }^{* *}$ other forms of myopathy or motor axonopathy could not be excluded; $\$$ description is reported in appendix 1 . 


\begin{tabular}{|c|c|c|c|c|c|c|c|}
\hline Authors & $\begin{array}{l}\text { Study design/ } \\
\text { Setting/aim }\end{array}$ & $\begin{array}{l}\text { N/followed/ } \\
\text { CIPNM type }\end{array}$ & Etiology & Follow-up & $\begin{array}{l}\text { Functional } \\
\text { measures/ } \\
\text { other }\end{array}$ & Other measures & Outcome \\
\hline \multirow[t]{2}{*}{$\begin{array}{l}\text { Nguyen The } \\
\mathrm{N} \text { et al }{ }^{44} \\
(2015)\end{array}$} & \multirow[t]{2}{*}{$\begin{array}{l}\text { Cohort, prospective } \\
\text { longitudinal } \\
\text { observational; } \\
\text { single center; } \\
\text { neurology; } \\
\text { incidence and } \\
\text { distribution of } \\
\text { CIP/CIM subtypes } \\
\text { and the evaluation } \\
\text { of the risk factors } \\
\text { and outcomes }\end{array}$} & $\begin{array}{l}\mathrm{N}=133 \mathrm{pts} \\
\mathrm{N}=73 \mathrm{pts} ; \\
\mathrm{CIP}=35 ; \\
\mathrm{CIM}=16 ; \\
\mathrm{CIP} / \mathrm{CIM}= \\
22 ; \\
\text { controls = } 60\end{array}$ & $\begin{array}{l}\text { medical } \\
\text { disorders }\end{array}$ & $3 \mathrm{mos}$ & none & $\begin{array}{l}\text { MRC; EMG; } \\
\text { ONLS }\end{array}$ & \multirow[t]{2}{*}{$\begin{array}{l}\text { At the end of the } \\
\text { follow-up duration } \\
\text { ( } 90 \text { days), } 31 \text { pts } \\
\text { with CIP/CIM were } \\
\text { evaluated: the } \\
\text { ONLS scores } \\
\text { improved but } \\
\text { remained } \\
\text { significantly } \\
\text { higher in } \\
\text { comparison to } \\
\text { non-CIP }(2.7 \text { vs } \\
0.8, p=0.015) ; \\
36(49 \%) \text { died }\end{array}$} \\
\hline & & & & & & & \\
\hline $\begin{array}{l}\text { Intiso D et } \\
\mathrm{al}^{45}(2017)\end{array}$ & $\begin{array}{l}\text { prospective cohort } \\
\text { study; single center, } \\
\text { neuro-rehabilitation } \\
\text { setting; functional } \\
\text { recovery in subjects } \\
\text { with sABI and } \\
\text { CIPNM }\end{array}$ & $\begin{array}{l}\mathrm{N}=36 ; \\
(27 \mathrm{M}, 9 \mathrm{~F}, \\
\text { mean age } \\
56.2 \pm 14.8 \\
\text { yrs }) \\
\mathrm{CIP}=36 ; \\
\mathrm{N}=75 \\
\text { controls } \\
\text { (sABI) }\end{array}$ & $\begin{array}{l}\text { patients with } \\
\text { sABI }\end{array}$ & $\begin{array}{l}107 \text { days } \\
(65-146)\end{array}$ & $\begin{array}{l}\text { LCF, DRS, } \\
\text { GOS, mRS }\end{array}$ & LOS & $\begin{array}{l}\text { The magnitude of } \\
\text { these } \\
\text { improvements } \\
\text { was different } \\
\text { between the } \\
\text { groups, showing } \\
\text { that patients with } \\
\text { sABI only had a } \\
\text { better } \\
\text { improvement than } \\
\text { those with } \\
\text { CIPNM+SABI for } \\
\text { mRS and DRS at } \\
\text { discharge. }\end{array}$ \\
\hline
\end{tabular}

Legend: $A D L=$ activity daily living; $D R S$ = disability rating scale; $G O S$ = Glasgow outcome scale; dmCMAP= direct muscle stimulation; ES= electrophysiological studies; FIM = Functional independence measure; ICF = International Classification of Functioning, Disability and Health; ICUAP = Intensive Care Unit acquired paresis; IPA = Impact on Participation and Autonomy questionnaire; LCF = Levels of Cognitive Functioning; LOS = length of stay; MRC = Medical Research Council scale; $m R S=$ modified Rankin Scale; ODSS = Overall Disability Sum score; ONLS = Overall Neuropathy Limitations Scale; neCMAP= nerve stimulation; RLOS = rehabilitation length of stay; RMI = Rivermead mobility index; $\mathrm{sABI}=$ severe acquired brain injury; SCI = spinal cord injury; SIP-68 = Sickness Impact Profile; SF 36= Short Form 36 questionnaire; TBI = traumatic brain injury; CRS-R = Coma Recovery Scale-Revised; GOS-E = Glasgow Outcome Scale-Expanded; FOIS = Functional Oral Intake Scale

${ }^{\circ}$ number of patients who had polyneuropathy to EMG; $£$ the sample included children and CIP was not defined; *including 12 patients described in a precedent paper; ${ }^{* *}$ other forms of myopathy or motor axonopathy could not be excluded; $\$$ description is reported in appendix 1 . 


\begin{tabular}{|c|c|c|c|c|c|c|c|}
\hline Authors & $\begin{array}{l}\text { Study design/ } \\
\text { Setting/aim }\end{array}$ & $\begin{array}{l}\text { N/followed/ } \\
\text { CIPNM type }\end{array}$ & Etiology & Follow-up & $\begin{array}{l}\text { Functional } \\
\text { measures/ } \\
\text { other }\end{array}$ & Other measures & Outcome \\
\hline $\begin{array}{l}\text { Cunningham } \\
\text { CJB et } \mathrm{al}^{46} \\
(2018)\end{array}$ & $\begin{array}{l}\text { prospective } \\
\text { observational; } \\
\text { case-control; } \\
\text { rehabilitation } \\
\text { setting; } \\
\text { prevalence of } \\
\text { CIPNM in } \\
\text { rehabilitative } \\
\text { setting and } \\
\text { impact of CIPNM } \\
\text { on function }\end{array}$ & $\begin{array}{l}\mathrm{N}=23,(19 \mathrm{M}, \\
4 \mathrm{~F}, \mathrm{mean} \text { age } \\
43.6 \pm 14.7) ; \\
\mathrm{CIP}=16 ; \\
\mathrm{CIM}=2 ; \\
\mathrm{CIP} / \mathrm{CIM}=5 \\
\text { controls }=10\end{array}$ & $\begin{array}{l}\text { medical } \\
\text { disorders, } \\
12 \text { pts had SCI } 2 \\
\text { pts stroke and } \\
\text { one TBI }\end{array}$ & $1 \mathrm{yr}$ & $\begin{array}{l}\text { FIM; FIM gain } \\
\text { and FIM } \\
\text { efficiency }\end{array}$ & $\begin{array}{l}\text { EMG/ES; } \\
\text { rehabilitation } \\
\text { length of stay } \\
\text { (RLOS), and } \\
\text { discharge } \\
\text { disposition }\end{array}$ & $\begin{array}{l}\text { FIM score: } 64.1 \\
\text { and } 89.7 \text { at } \\
\text { admission; } 78.4 \\
\text { and } 94.6 \text {, at } \\
\text { discharge in pts } \\
\text { with CIPNM and } \\
\text { without CIPNM, } \\
\text { respectively. The } \\
\text { gains in FIM } \\
\text { scores and RLOS } \\
\text { were greater, } \\
\text { leading to similar } \\
\text { FIM efficiency } \\
\text { (FIM points } \\
\text { gained/day of } \\
\text { rehabilitation) } \\
\text { compared with } \\
\text { those without } \\
\text { CIPNM (only for } \\
13 \text { pts). Those } \\
\text { with CIPNM were } \\
\text { less likely to be } \\
\text { discharged } \\
\text { directly home } \\
\text { (57\% versus } 90 \% \text { ). } \\
\text { At } 1 \text { year, recovery } \\
\text { was seen in } 80 \% \\
\text { of those with CIM } \\
\text { and } 55 \% \text { of those } \\
\text { with CIM/CIP }\end{array}$ \\
\hline $\begin{array}{l}\text { Symeonidou } \\
\text { Z et al }{ }^{47} \\
(2019)\end{array}$ & $\begin{array}{l}\text { multicenter; } \\
\text { retrospective } \\
\text { observational; } \\
\text { rehabilitation } \\
\text { setting; functional } \\
\text { recovery }\end{array}$ & $\begin{array}{l}\mathrm{N}=28 \text { pts }(19 \\
\mathrm{M}, 9 \mathrm{~F}, \text { mean } \\
\text { age } \\
53.6 \pm 14.5) \\
\mathrm{CIP}=28\end{array}$ & $\begin{array}{l}\text { medical } \\
\text { disorders; } \\
\text { cerebral or } \\
\text { spinal cord } \\
\text { injury or stroke } \\
\text { were excluded }\end{array}$ & $\begin{array}{l}109.4 \pm 70.7 \\
\text { days }\end{array}$ & $\begin{array}{l}\text { Barthel Index; } \\
\text { ADL }\end{array}$ & $\begin{array}{l}\text { MRC, sensory } \\
\text { examination }\end{array}$ & $\begin{array}{l}\text { Mean Barthel } \\
\text { score at } \\
\text { admission and } \\
\text { discharge } \\
\text { improved } \\
\text { significantly ( } 15.3 \\
\pm 9.1 \text { vs } 63.6 \pm \\
21.6, \text { p<0.05); } \\
3(10.7 \%) \text { pts had } \\
\text { complete recovery; } \\
13(46.4 \%) \text { pts } \\
\text { incomplete } \\
\text { recovery (Barthel } \\
\text { score } 65-80) ; 5 \text { pts } \\
\text { had severe } \\
\text { disability, at } \\
\text { discharge }\end{array}$ \\
\hline
\end{tabular}

Legend: $A D L$ = activity daily living; $D R S$ = disability rating scale; GOS = Glasgow outcome scale; dmCMAP= direct muscle stimulation; ES= electrophysiological studies; FIM = Functional independence measure; ICF = International Classification of Functioning, Disability and Health; ICUAP = Intensive Care Unit acquired paresis; IPA = Impact on Participation and Autonomy questionnaire; LCF = Levels of Cognitive Functioning; LOS = length of stay; MRC = Medical Research Council scale; $m R S=$ modified Rankin Scale; ODSS = Overall Disability Sum score; ONLS = Overall Neuropathy Limitations Scale; neCMAP= nerve stimulation; RLOS = rehabilitation length of stay; RMI = Rivermead mobility index; $\mathrm{sABI}=$ severe acquired brain injury; SCI = spinal cord injury; SIP-68 = Sickness Impact Profile; SF 36= Short Form 36 questionnaire; TBI = traumatic brain injury; CRS-R = Coma Recovery Scale-Revised; GOS-E = Glasgow Outcome Scale-Expanded; FOIS = Functional Oral Intake Scale

${ }^{\circ}$ number of patients who had polyneuropathy to EMG; $£$ the sample included children and CIP was not defined; *including 12 patients described in a precedent paper; ${ }^{* *}$ other forms of myopathy or motor axonopathy could not be excluded; $\$$ description is reported in appendix 1 . 


\begin{tabular}{|c|c|c|c|c|c|c|c|}
\hline Authors & $\begin{array}{l}\text { Study design/ } \\
\text { Setting/aim }\end{array}$ & $\begin{array}{l}\text { N/followed/ } \\
\text { CIPNM type }\end{array}$ & Etiology & Follow-up & $\begin{array}{l}\text { Functional } \\
\text { measures/ } \\
\text { other }\end{array}$ & Other measures & Outcome \\
\hline $\begin{array}{l}\text { Hakiki B et } \\
\text { al }^{48}(2021)\end{array}$ & $\begin{array}{l}\text { Single; retrospective } \\
\text { observational; } \\
\text { rehabilitation } \\
\text { setting; functional } \\
\text { recovery }\end{array}$ & $\begin{array}{l}\mathrm{N}=224 \mathrm{pts} ; \\
(81(36 \%) \\
\text { females, age } \\
68.73) ; \\
\text { CIPNM = } 119\end{array}$ & $\begin{array}{l}\text { patients with } \\
\text { SABI }\end{array}$ & $3.8 \mathrm{mos}$ & $\begin{array}{l}\text { CRS-R; FIM; } \\
\text { GOS-E; FOIS }\end{array}$ & ENG/EMG & $\begin{array}{l}\text { All patients gained } \\
\text { functional } \\
\text { improvement at } \\
\text { discharge: } P< \\
.001 \text { for FOIS, FIM } \\
\text { and GOS-E), but } \\
\text { those with a } \\
\text { concomitant } \\
\text { CIPNM achieved } \\
\text { significantly lower } \\
\text { scores ( } \text { < }<001 \\
\text { for FIM and GOS- } \\
\text { E). The CIPNM } \\
\text { absence was } \\
\text { associated with a } \\
\text { higher probability } \\
\text { to achieve } \\
\text { functional } \\
\text { autonomy }\end{array}$ \\
\hline $\begin{array}{l}\text { Legend: ADL } \\
\text { electrophysi } \\
\text { ICUAP = Inte } \\
\text { LOS = lengtt } \\
\text { Neuropathy } \\
\text { acquired bra } \\
\text { CRS-R = Cor }\end{array}$ & $\begin{array}{l}\text { activity daily living; DR } \\
\text { gical studies; FIM = Fu } \\
\text { ve Care Unit acquired p } \\
\text { f stay; MRC = Medical } \\
\text { nitations Scale; neCMA } \\
\text { injury; SCI = spinal cor } \\
\text { Recovery Scale-Revise }\end{array}$ & $\begin{array}{l}S=\text { disability rati } \\
\text { nctional indepen } \\
\text { aresis; IPA = Imp } \\
\text { Research Council } \\
P=\text { nerve stimula } \\
\text { injury; SIP-68 = } \\
\text { d; GOS-E = Glasg }\end{array}$ & $\begin{array}{l}\text { g scale; GOS = } \\
\text { ence measure; I } \\
\text { ct on Participati } \\
\text { cale; mRS = mc } \\
\text { on; RLOS = reha } \\
\text { ickness Impact } \\
\text { W Outcome Sca }\end{array}$ & $\begin{array}{l}\text { gow outcom } \\
\text { Internationa } \\
\text { nd Autonom } \\
\text { d Rankin Sc } \\
\text { ation length } \\
\text { le; SF } 36=\text { S } \\
\text { panded; FOI }\end{array}$ & $\begin{array}{l}\text { scale; dmCMAP } \\
\text { Classification of } \\
\text { questionnaire; } \\
\text { e; ODSS = Over } \\
\text { stay; RMI = Riv } \\
\text { ort Form } 36 \text { que } \\
=\text { = Functional Or }\end{array}$ & $\begin{array}{l}\text { direct muscle stim } \\
\text { unctioning, Disabi } \\
\text { F = Levels of Cogn } \\
\text { Disability Sum scc } \\
\text { mead mobility ind } \\
\text { innaire; TBI = trau } \\
\text { Intake Scale }\end{array}$ & $\begin{array}{l}\text { lation; ES= } \\
\text { ty and Health; } \\
\text { tive Functioning; } \\
\text { re; ONLS = Overall } \\
\text { x; SABI = severe } \\
\text { natic brain injury; }\end{array}$ \\
\hline
\end{tabular}

\section{Functional outcomes}

Determining the functional outcome was the main purpose of 9 (31\%) studies [22-23, 40-43, 45, 47-48]. Overall, 70.3\% of subjects with CIPNM achieved a full recovery. Eighteen studies reported a percentage above $50 \%$ and among these, 10 showed that $75 \%$ of the sample (range $75-100$ ) reached a full recovery. Functional measures were variable and overall, 16 tools were used to evaluate recovery: the Barthel Index (39-41, 47), the FIM $[42,46,48]$, the mRS [41, 45], the ADL [47], the DRS [45], the GOS [45], GOS-E [48]; the CRS-R [48]; the Rivermead Motor Assessment Scale (RMA) (40), the functional disability, the Jebsen hand function test [40], the timed UP \& GO walking test [40], the global motor performance [22], the 6-min (expressed in meters) and 10-meters walking test (expressed in speed velocity) [42], the Overall Disability Sum score (ODSS) [43], and the Functional Health Status [23]. The Barthel Scale and the FIM were employed in 4 [39-41, 47] and 3 studies [42, 46, 48], respectively. The studies that used the Barthel scale showed contrasting findings. Of these, 2 prospective studies reported that $86.3 \%$ and $73.8 \%$ of the sample, respectively, achieved a good recovery (mean Barthel score $>90$ ) at follow-up $[39,41]$. Conversely, $10.7 \%$ and $31.2 \%$ of subjects made a full recovery in the remaining 2 studies [ 47 , 40], respectively. The studies that used the FIM showed a significant functional improvement. In this regard, Novak et al. reported that subjects with CIP had a mean FIM score of 78.7 \pm 24.12 and 103.3 \pm 20.5 , at admission and discharge, respectively $(p<0.001)$ [42]. Likewise, a prospective observational case control study by Cunningham et al. found that subjects with CIPNM had greater gains in FIM scores, leading to similar FIM efficiency (FIM points gained/day of rehabilitation), than subjects without CIPNM [46]. However, despite these seemingly functional improvements and similar discharge FIM scores, subjects with CIPNM were less likely to be discharged directly home (57\% versus $90 \%$ ).

Fourteen (48.2\%) studies did not have patient's global ability as primary end-point and did not use functional scales. Recovery was evaluated from a clinical point of view by neurological examination or on the basis of the improvement in muscle strength by the MCR scale. This measure was used in 11 studies [22-23, 28, 30, 36, 38, 40, 42-44,47]. The severity of muscle weakness was not correlated with the clinical and electrophysiological diagnosis, and there was no correlation between the degree of the nerve conduction and the clinical findings [22, 37].

\section{Long-term outcomes}

Seven studies (24.1\%) had a follow-up longer than 1 year, ranging from 2 to 8 years (Table 2 ) for a total of 173 (21.9\%) subjects, of whom 37 (21.3\%) died and 108 were followed. Overall 124, 43 and 6 subjects had CIP, CIM and CIP/CIM, respectively. Of followed subjects with CIPNM 96 (88.8\%) achieved a full recovery. Almost all studies included small samples characterized by case series including 7 to 22 patients, and only 2 studies had greater samples consisting of 49 [35] and 42 subjects [41], respectively. Furthermore, the investigation of long-term functional outcomes in subjects with CIPNM as main purpose was addressed only in 1 of these 7 studies [41]. This study had a mixed sample and included 42 patients with different etiology of ICU admission and showed that $73.8 \%$ of the entire sample of patients made a good recovery, as shown by the functional measures at follow-up: mean Barthel and median mRS score of $86.7 \pm 15.9$ and 1 (IQR: $0-5$ ), respectively. The differentiation between the types of CIPNM was performed only in 2 studies $[35,41]$. Both studies showed that subjects with CIP/CIM had the worst outcome. Functional measures were employed only in 2 studies that used the Barthel scale $[39,41]$ and the mRS [41]. Both studies had a long follow-up of 3.5 and 5 years, respectively, and a good

Page 13/22 
recovery was detected in a high percentage of subjects. Five (71.4\%) studies did not use proper scales, and the recovery was evaluated by motor improvement, such as the ability to walk without support or aid.

Table 2

Long-term functional outcome in subjects with critical illness polyneuropathy and myopathy

\begin{tabular}{|c|c|c|c|c|c|}
\hline Authors & $\begin{array}{l}\text { N/followed/ } \\
\text { CIPNM type }\end{array}$ & Follow-up & $\begin{array}{l}\text { Functional } \\
\text { measures }\end{array}$ & $\begin{array}{l}\text { Other } \\
\text { measures }\end{array}$ & Outcome \\
\hline $\begin{array}{l}\text { Zochodne } \\
\text { DW et } \\
\text { al }^{24} \\
(1987)\end{array}$ & $\begin{array}{l}\mathrm{N}=19 ; \\
(9 \mathrm{M}, 10 \mathrm{~F}, \\
\text { mean age } \\
64) ; \\
\mathrm{CIP}=19\end{array}$ & $\begin{array}{l}10 \text { mos-2 } \\
\text { yrs }\end{array}$ & none & $\begin{array}{l}\text { EMG, } \\
\text { histological } \\
\text { examination }\end{array}$ & $\begin{array}{l}8(41.1 \%) \text { pts showed good functional recovery; of these } 6 \text { pts had EMG } \\
\text { improvement within } 3 \text { months. One pt had mild distal limb weakness at } \\
12 \text { wks and one had mildly weak dorsiflexion of right foot, at } 2 y r s ; 11 \\
(58 \%) \text { pts died }\end{array}$ \\
\hline $\begin{array}{l}\text { Coronel B } \\
\text { et } \mathrm{al}^{25} \\
(1990)\end{array}$ & $\begin{array}{l}N=15 / 4 \\
(12 \mathrm{M}, 3 \mathrm{~F}, \\
\text { mean age } \\
47) ; \\
\mathrm{CIP}=15\end{array}$ & $4-8$ yrs & none & $\begin{array}{l}\text { EMG, muscle } \\
\text { biopsies }\end{array}$ & $\begin{array}{l}3 \text { pts: } 2 \text { pts had persisting dysesthesia; one needing assistance to sit } \\
\text { and walk } \\
\text { death: } 5 \text { pts }(33 \%)\end{array}$ \\
\hline $\begin{array}{l}\text { Hund EF } \\
\text { et al }{ }^{33} \\
(1996)\end{array}$ & $\begin{array}{l}N=7 \\
(3 M, 4 F)\end{array}$ & $\begin{array}{l}3 \text { mos to } \\
3.5 \text { yrs }\end{array}$ & none & $\begin{array}{l}\text { EMG; muscle } \\
\text { and nerve } \\
\text { biopsy ( } 3 \\
\text { pts) }\end{array}$ & $\begin{array}{l}2(28.5 \%) \text { pt gained complete recovery; } \\
3(42.8 \%) \text { pts showed disability due to CNS lesions; } 2 \text { pts died }\end{array}$ \\
\hline $\begin{array}{l}\text { Lacomis } \\
\text { D. et al }{ }^{35} \\
(1997)\end{array}$ & $\begin{array}{l}\mathrm{N}=92 \\
\mathrm{~N}=49 \\
\mathrm{CIM}=37 \\
\mathrm{CIP}=12\end{array}$ & $\begin{array}{l}12-60 \\
\text { mos }\end{array}$ & none & $\begin{array}{l}\text { EMG; muscle } \\
\text { biopsies ( } 22 \\
\text { pts) }\end{array}$ & $\begin{array}{l}25(51 \%) \text { pts had complete functional recovery: } 17 \text { pts were ambulatory } \\
\text { within } 4 \text { months and } 8 \text { pts within } 4-12 \text { months; } \\
7 \text { pts showed incomplete functional recovery: } 4 \text { pts remained non } \\
\text { ambulatory and } 3 \text { remained dependent on the ventilator; } 16 \text { pts died }\end{array}$ \\
\hline $\begin{array}{l}\text { de Sèze } \\
\text { M. et al } \\
(2000)\end{array}$ & $\begin{array}{l}\mathrm{N}=19, \text { only } \\
\text { CIP }(14 \mathrm{M} 5 \\
\text { F; mean age } \\
55,9 \text { yrs })\end{array}$ & $2 \mathrm{yrs}$ & None & $\begin{array}{l}\text { MRC; } \\
\text { sensory } \\
\text { findings }\end{array}$ & $\begin{array}{l}11(57.8 \%) \text { patients recovered completely; } \\
4(21 \%) \text { patients remained quadriplegic; } 2 \text { patients remained } \\
\text { quadriparetic; } 2 \text { pts died }\end{array}$ \\
\hline $\begin{array}{l}\text { Fletcher } \\
\text { S.N. et } \\
\text { al } \\
(2003)\end{array}$ & $\begin{array}{l}N=22 \\
\text { CIP= 22; } \\
62 \text { yrs }(45- \\
78)\end{array}$ & $\begin{array}{l}3.5 \text { yrs } \\
\text { (range, } \\
12-57 \\
\text { mos) }\end{array}$ & $\begin{array}{l}\text { Barthel } \\
\text { Index }\end{array}$ & $\begin{array}{l}\text { neurologic } \\
\text { examination; } \\
\text { EMG }\end{array}$ & $\begin{array}{l}19(86.3 \%) \text { pts had full recovery (BI score } 95-100) ; 2 \text { pts incomplete } \\
\text { recovery (BI score } 85) ; 1 \text { pts severe disabled; } 95 \% \text { patients had EMG } \\
\text { evidence of chronic partial denervation. }\end{array}$ \\
\hline $\begin{array}{l}\text { Intiso D et } \\
\text { al }^{41} \\
(2011)\end{array}$ & $\begin{array}{l}\mathrm{N}=42(23 \mathrm{M}, \\
19 \mathrm{~F}, \text { mean } \\
\text { age } 58.4 \pm \\
13.9) ; \\
\text { CIP= } 30 \text { pts } \\
\mathrm{CIM}=6 \mathrm{pts} \\
\mathrm{CIP} / \mathrm{CIM}=6 \\
\text { pts }\end{array}$ & $\begin{array}{l}5 \text { yrs; } \\
\text { mean } \\
31.7 \pm 15.8 \\
\text { months }\end{array}$ & $\begin{array}{l}\text { Barthel } \\
\text { scale and } \\
\text { mRS }\end{array}$ & $\begin{array}{l}\text { SF-36 } \\
\text { questionnaire }\end{array}$ & $\begin{array}{l}31(73.8 \%) \text { pts ( } 24 \text { pts with just CIPNM and } 7 \text { pts with CIPNM and CNS } \\
\text { involvement) gained good recovery: mean Barthel of } 86.7 \pm 15.9(\mathrm{P}< \\
0.001) \text {, and the median mRS of } 1 \text { (IQR: } 0-3) \text {, respectively, at follow-up } \\
\text { (mean } 31.7 \pm 15.8 \text { months). }\end{array}$ \\
\hline
\end{tabular}

\section{CIPNM type and outcome}

The differentiation between the types of CIPNM was performed in 7 (25\%) studies [6, 22-23, 35, 41, 44, 46]. Among these, 3 investigations concerning the long-term functional outcome reported that the CIM type had a better prognosis and an earlier recovery than CIP/CIM [22-23, 41]. In detail, Koch et al. enrolled a cohort of 26 subjects consisting of 11 and 8 patients with CIP/CIM and CIM, respectively, whereas the remaining 7 were controls. After 1 year of follow up, in 7 (87.5\%) and 6 (54.5\%) patients with CIM and CIP/CIM, respectively, a return to normal physical capacity and a normal EMG were observed. Furthermore, $50 \%$ of CIM patients recovered within 3 months, returning to a normal or at least a sufficient physical capacity to resume daily life activities. On the other hand, 5 (45.5\%) patients with CIP/CIM had a partial recovery and abnormal electrophysiological findings. Guarneri et al. reported the long-term recovery of 15 patients with CIPNM and, of these, 4,6 and 3 patients had CIP, CIM and CIP/CIM, respectively, whereas 2 subjects were undetermined (Table 1). Five subjects with CIM recovered within 6 months, whereas the presence of CIP alone or in addition to CIM was associated with a more delayed recovery between 6 and 12 months, and more than $50 \%$ of those individuals had persistent deficits at 1 year of followup [22]. Likewise, the study by Intiso et al. reported that CIM patients had a better recovery than subjects with CIP or CIP/CIM and did not show 
differences in their health status compared to the Italian normative data. Of the remaining 4 studies, 3 did not report data on the recovery of CIPNM types, since CIPNM subjects were evaluated in comparison to controls independently of the type of CIPNM [6, 44, 46], and the last one had severe limitations despite the differentiation between the types of CIPNM, since other forms of myopathy or motor axonopathy could not be excluded [35].

\section{Discussion}

The present review detected that $70.3 \%$ of a large number subjects with CIPNM could achieve a full recovery. This finding is similar to that obtained from a previous review by Latronico et al., who reported that $68.8 \%$ of patients made a complete recovery [21]. Furthermore, a higher percentage of $88.8 \%$ gained full recovery at long term follow-up. However, because of strong limitations of studies that had small samples and were widely variable in aim, methodology design, case mix, and outcome measures, the finding should be considered with caution. The investigation of the functional outcome was the main purpose in $31 \%$ of the studies analyzed; in addition, a variety of measures was employed, and only 7 ( $24.1 \%$ ) studies used a proper functional scale such as the Barthel scale and the FIM.

Of great importance, immediately after the rehabilitation treatment, is the overall health status of the patients, culminating in a return to active daily living, socialization and participation. The ICF recommends a new approach to evaluate disabled people, which is based on a holistic model in which activities and participation represent essential aspects. In this respect, only 2 studies investigated the outcome according to the ICF, but because of the small size of the samples and the limited duration of the follow-ups, it was not possible to draw definitive conclusions [40, 42]. Most studies (71.4\%) evaluated recovery on the basis of the motor improvement and the ability to walk without support, but no data was reported on the functionality in activity daily living. Furthermore, though the present review retrieved a larger number of 788 patients with CIPNM, those who had long term follow-up for more than 1 year were only $173(21.9 \%)$ subjects.

Over two decades, until year 2000, the main purpose of investigators was to characterize the new clinical phenomenon. There were no studies having patient's global ability and quality of life as primary end-points. The majority of these studies reported the outcome in terms of complete or incomplete recovery (30-31, 35-36), motor performance [30-31] and ambulatory activity [6, 30,35]. The outcome was also addressed, but it was generically labeled as "full recovery" or based on the achievement of the motor ability, particularly of ambulation without support or aid. A proper functional scale quantifying disability and evaluating the patients' health status and quality of life was not employed. The evaluation of the functional outcome was performed on the neurological examination or on the improvement of the muscle strength by the MCR scale. Clinical recovery was considered complete if patients had an MRC grade of $4 / 5$ in all muscles $(22,30)$. Although this scale has been recently demonstrated to be an important predictor of death and of a worse five-year survival [56], it was designed and validated to quantify muscle strength impairment but not in relation to functional abilities. Most studies were performed by ICU specialists, and this may explain the methodological approach used. In this respect, ICU specialists might prefer to assess muscle weakness and to diagnose early CIPNM during the ICU stay, since this disorder could be a severe complication for the weaning of mechanical ventilation [57-58] and could delay ICU discharge.

Some authors suggested that patients with the CNS injury might likewise develop critical illness polyneuropathy and, consequently, recovery might be hampered by the CNS damage $(33,35)$; however, only $4(14.2 \%)$ studies $[38,40,43,47]$ excluded subjects with the CNS damage to avoid confounding findings on the outcome. On the other hand, 2 studies were designed to investigate recovery in subjects with sABI and coexistent CIPNM [45]. The authors reported that patients with SABI and CIPNM achieved a good recovery, but the magnitude of these improvements was better in the subjects with $\mathrm{SABI}$ alone. However, it is still not clear if the residual disability in these subjects is due predominantly to CIPNM, to CNS damage or to both disorders. Likewise, the effect that each disease might have on the course of the disability still needs to be clarified. People with both disorders might require different rehabilitation approaches and strategies.

\section{Outcome of the types of CIPNM}

It has been suggested that subjects with CIM have a better prognosis, reaching early and full recovery, than subjects with CIP or CIP/CIM, but this finding was reported only in 3 studies including a total of 20 patients [22-23, 41]. Therefore, it is not possible to achieve a definitive conclusion about this matter due to the very small number of investigated subjects. Several difficulties hamper and make it hard to make real and objective comments on this issue. Some of these difficulties are the lack of unique and shared definitions of muscle weakness that may affect ICU subjects as well as an insufficient differentiation between the types of CIPNM. Today, a wide range of definitions are still being used including ICUAW, CIP, CIM or CIP/CIM, and this aspect can complicate the analysis, and the results of the studies may be consequently biased. Most investigations considered this disorder as a single entity, therefore it is not possible to exclude that different forms of CIPNM were present in the sample of the studies investigating only subjects with CIP. Furthermore, although EMG is able to differentiate between the subtypes of CIPNM, it does not allow the quantification of the muscle impairment and the related disabilities, and no electrophysiological exams or imaging have helped to solve this point.

\section{Limitations}

The present study has limitations that must be acknowledged. This is a review of the literature having the aim to discuss the recovery and the longterm functional outcome of CIPNM subjects. Cohort studies, case series as well as functional measurements and follow-up were highly heterogeneous regarding the functional outcome. A further limitation concerns the recovery of subjects with CIPNM; such limitation is due to the paucity of trials focusing on rehabilitative interventions $[41-42,59]$. Even if this issue is beyond the scope of the present review, the majority of the studies analyzed 
did not define whether the subjects followed any kind of rehabilitation treatment after hospital discharge. Currently, apart from the early neuromuscular electrical stimulation that might prevent ICUAW and improve the quality of life by enhancing muscle strength in ICU patients [59], no definitive studies have evaluated the effects of rehabilitation programs in inpatient or outpatient settings in this population [60]. Therefore, several questions remain unanswered and further research should be carried out on this matter.

\section{Suggestions and implications for the future}

Given the protean aspects of CIPNM, a closer collaboration as well as a more active participation of multiple specialists and experts has been suggested [61]. In particular, ICU specialists, neurologists and physiatrists should collaborate more to properly evaluate and follow these subjects. Specialists who manage CIPNM patients should adopt unique and shared terminology and definitions, and future studies should be planned considering the following aspects:

- the aim should be focused on functional recovery; the methodology design should include a large sample of patients, proper functional measures and defined long-term follow-up;

- differentiation between the types of CIPNM;

- rehabilitation interventions and their effect on functional outcome and quality of life, given that few studies have evaluated the effects of rehabilitation programs in this population.;

- Occurrence and recovery of CIPNM in subjects with CNS damage should be investigated through dedicated studies.

\section{Conclusion}

A percentage of $70.3 \%$ of survivor subjects with CIPNM could achieve a full recovery and a higher percentage was detected at long term follow-up. However, the quality of the published studies due to short follow-ups, and the absence of clearly defined outcome measures did not allow definitive conclusions. A close collaboration between specialists and proper planned research in this field are needed to answer the unsolved questions.

\section{Abbreviation}

$\mathrm{ADL}$ = activity of daily living; $\mathrm{DRS}$ = disability rating scale; GOS = Glasgow outcome scale; dmCMAP= direct muscle stimulation; $\mathrm{ES}=$ electrophysiological studies; FIM = Functional independence measure; ICF = International Classification of Functioning, Disability and Health; ICUAP= Intensive Care Unit acquired paresis; IPA = Impact on Participation and Autonomy questionnaire; LCF = Levels of Cognitive Functioning; LOS = length of stay; MRC = Medical Research Council scale; $m R S=$ modified Rankin Scale; ODSS = Overall Disability Sum score; ONLS = Overall Neuropathy Limitations Scale; neCMAP = nerve stimulation; RLOS = rehabilitation length of stay; RMI = Rivermead mobility index; $\mathrm{SABI}=$ severe acquired brain injury; SCI = spinal cord injury; SIP-68 = Sickness Impact Profile; SF 36= Short Form 36 questionnaire; TBI = traumatic brain injury; CRS-R = Coma Recovery Scale-Revised; GOS-E = Glasgow Outcome Scale-Expanded; FOIS = Functional Oral Intake Scale

\section{Declarations}

\section{Ethics approval and consent to participate}

"not applicable"

All authors consent for publication.

The datasets used and/or analysed during the current study available from the corresponding author on reasonable request.

\section{Conflict of interest statement}

The authors declare that there is no conflict of interest.

\section{Funding}

The authors received no financial support for the research, authorship, and/or publication of this article.

\section{Author Contributions}

Conceptualization: D.I., F.DR.; Data extraction: M.C, MB; MTG; MG; Manuscript preparation: D.I., M.B., FDR; Review and revision: DI, FDR. All authors have read and agreed to the version of the manuscript.

\section{Acknowledgements}

"not applicable" 


\section{References}

1. Stevens RD, Marshall SA, Cornblath DR, Hoke A, Needham DM, de Jonghe B, Ali NA, Sharshar T. A framework for diagnosing and classifying intensive care unit-acquired weakness. Crit Care Med. 2009;37(10 Suppl):S299-308.

2. Op de Coul AA, Verheul GA, Leyten AC, et al. Critical illness polyneuromyopathy after artificial respiration. Clin Neurol Neurosurg. 1991;93:27-33

3. Bednarik J, Lukas Z, Vondracek P. Critical illness polyneuromyopathy: the electrophysiological components of a complex entity. Intensive Care Med 2003;29(9):1505-14

4. Bolton CF, Gilbert JJ, Hahn AF, Sibbald WJ (1984). Polyneuropathy in critically ill patients. J Neurol Neurosurg Psychiatry 1984;47(11):1223-31.

5. Lefaucheur JP, Nordine T, Rodriguez P, Brochard L. Origin of ICU acquired paresis determined by direct muscle stimulation. J Neurol Neurosurg Psychiatry 2006; 77(4):500-6.

6. Kerbaul F, Brousse M, Collart F, Pelissier JF, Planche D, Fernandez C, Gouin F, Guidon C. Combination of histopathological and electromyographic patterns can help to evaluate functional outcome of critical ill patients with neuromuscular weakness syndromes. Crit Care 2004; 8(6):R358-66

7. Hough CL, Steinberg KP, Taylor Thompson B, Rubenfeld GD, Hudson LD. Intensive care unit-acquired neuromyopathy and corticosteroids in survivors of persistent ARDS. Intensive Care Med. 2009; 35:63-68.

8. de Jonghe B, Lacherade JC, Sharshar T, Outin H. Intensive care unit-acquired weakness: risk factors and prevention. Crit Care Med. 2009; 37:S309S315.

9. Stevens RD, Dowdy DW, Michaels RK, Mendez-Tellez PA, Pronovost PJ, Needham DM. Neuromuscular dysfunction acquired in critical illness: a systematic review. Intensive Care Med. 2007; 33:1876-91.

10. Fan E, Cheek F, Chlan L et al. An official American Thoracic Society Clinical Practice guideline: the diagnosis of intensive care unit-acquired weakness in adults. Am J Respir Crit Care Med. 2014; Dec 15;190(12):1437-46.

11. Bednarík J, Vondracek P, Dusek L, Moravcova E, Cundrle I. Risk factors for critical illness polyneuromyopathy. J Neurol. 2005; 252(3):343-51.

12. Nanas S, Kritikos K, Angelopoulos E, Siafaka A, Tsikriki S, Poriazi M, Kanaloupiti D, Kontogeorgi M, Pratikaki M, Zervakis D, Routsi C, Roussos C. Predisposing factors for critical illness polyneuromyopathy in a multidisciplinary intensive care unit. Acta Neurol Scand 2008; 118(3):175-81.

13. Hermans G, De Jonghe B, Bruyninckx F, Van den Berghe G. Interventions for preventing critical illness polyneuropathy and critical illness myopathy. Cochrane Database Syst Rev 2014; 30;1:CD006832.

14. Shepherd SJ, Newman R, Brett SJ, Griffith DM. Enhancing Rehabilitation after Critical Illness Programme Study Investigators. Pharmacological therapy for the prevention and treatment of weakness after critical illness: A systematic review. Crit Care Med 2016; 44(6):1198-205.

15. Zayed Y, Kheiri B, Barbarawi M, Chahine A, Rashdan L, Chintalapati S, Bachuwa G, Al-Sanouri I. Effects of neuromuscular electrical stimulation in critically ill patients: A systematic review and meta-analysis of randomised controlled trials. Aust Crit Care 2020; 33(2):203-210.

16. Anekwe DE, Biswas S, Bussières A, Spahija J. Early rehabilitation reduces the likelihood of developing intensive care unit-acquired weakness: a systematic review and meta-analysis. Physiotherapy 2020; Jun;107:1-10

17. Zhang L, Hu W, Cai Z, Liu J, Wu J, Deng Y, Yu K, Chen X, Zhu L, Ma J, Qin Y. Early mobilization of critically ill patients in the intensive care unit: A systematic review and meta-analysis. PLoS One 2019; Oct 3;14(10):e0223185.

18. Hermans G, Van den Berghe G. Clinical review: intensive care unit acquired weakness. Crit Care 2015; 5;19(1):274.

19. Piva S, Fagoni N, Latronico N. Intensive care unit-acquired weakness: unanswered questions and targets for future research. F1000Res 2019 , Apr 17;8:F1000 Faculty Rev-508

20. Vanhorebeek I, Latronico N, Van den Berghe G. ICU acquired weakness. Intensive Care Med, 2020; Apr;46(4):637-653.

21. Latronico N, Shehu I, Seghelini E. Neuromuscular sequelae of critical illness. Curr Opin Crit Care 2005; 11(4):381-90.

22. Guarneri B, Bertolini G, Latronico N. Long-term outcome in patients with critical illness myopathy or neuropathy: the Italian multicentre CRIMYNE study. J Neurol Neurosurg Psychiatry 2008; 79(7):838-41.

23. Koch S, Wollersheim T, Bierbrauer J, Haas K, Mörgeli R, Deja M, Spies CD, Spuler S, Krebs M, Weber-Carstens S. Long-term recovery In critical illness myopathy is complete, contrary to polyneuropathy. Muscle Nerve 2014; 50(3):431-6

24. Zochodne DW, Bolton CF, Wells GA, et al. Critical illness polyneuropathy. A complication of sepsis and multiple organ failure. Brain 1987;110:819841.

25. Coronel B, Mercatello A, Couturier JC, et al. Polyneuropathy: potential cause of difficult weaning. Crit Care Med 1990; 18:486-489.

26. Witt NJ, Zochodne DW, Bolton CF, et al. Peripheral nerve function in sepsis and multiple organ failure. Chest 1991; 99:176-184.

27. Rossiter A, Souney PF, McGowan S, et al. Pancuronium-induced prolonged neuromuscular blockade. Crit Care Med 1991; $19: 1583-1587$.

28. Gooch JL, Moore MH, Ryser DK. Prolonged paralysis after neuromuscular junction blockade: case reports and electrodiagnostic findings. Arch Phys Med Rehabil 1993; 74(9):1007-11.

29. Giostra E, Magistris MR, Pizzolato G, et al. Neuromuscular disorder in intensive care unit patients treated with pancuronium bromide. Occurrence in a cluster group of seven patients and two sporadic cases, with electrophysiologic and histologic examination. Chest 1994; 106:210-220.

30. Leijten FS, Harinck-de Weerd JE, Poortvliet DC, et al. The role of polyneuropathy in motor convalescence after prolonged mechanical ventilation. JAMA 1995; 274:1221-1225.

Page $17 / 22$ 
31. Latronico N, Fenzi F, Recupero D, Guarneri B, Tomelleri G, Tonin P, De Maria G, Antonini L, Rizzuto N, Candiani A. Critical illness myopathy and neuropathy. Lancet 1996; 347(9015):1579-82.

32. Berek K, Margreiter J, Willeit J, et al. Polyneuropathies in critically ill patients: a prospective evaluation. Intensive Care Med 1996; $22: 849-855$.

33. Hund EF, Fogel W, Krieger D, et al. Critical illness polyneuropathy: clinical findings and outcomes of a frequent cause of neuromuscular weaning failure. Crit Care Med 1996; 24:1328-1333.

34. Campellone JV, Lacomis D, Kramer DJ, et al. Acute myopathy after liver transplantation. Neurology 1998; 50:46-53.

35. Lacomis D, Petrella JT, Giuliani MJ. Causes of neuromuscular weakness in the intensive care unit: a study of ninety-two patients. Muscle Nerve 1998; 21:610-617.

36. de Seze M, Petit H, Wiart L, et al. Critical illness polyneuropathy. A 2-year follow-up study in 19 severe cases. Eur Neurol 2000; 43:61-69.

37. Zifko UA. Long-term outcome of critical illness polyneuropathy. Muscle Nerve 2000; Suppl 9:S49-S52.

38. De Jonghe B, Sharshar T, Lefaucheur JP, et al. Paresis acquired in the intensive care unit: a prospective multicenter study. JAMA 2002; 288:28592867.

39. Fletcher SN, Kennedy DD, Ghosh IR, et al. Persistent neuromuscular and neurophysiologic abnormalities in long-term survivors of prolonged critical illness. Crit Care Med 2003; 31:1012-1016.

40. van der Schaaf M, Beelen A, Dongelmans DA, Vroom MB, Nollet F. Poor functional recovery after a critical illness: a longitudinal study. J Rehabil Med 2009; 41(13):1041-8

41. Intiso D, Amoruso L, Zarrelli M, Pazienza L, Basciani M, Grimaldi G, larossi A, Di Rienzo F. Long-term functional outcome and health status of patients with critical illness polyneuromyopathy. Acta Neurol Scand 2011; 123(3):211-9

42. Novak P, Vidmar G, Kuret Z, Bizovičar N. Rehabilitation of critical illness polyneuropathy and myopathy patients: an observational study. Int J Rehabil Res 2011; 34(4):336-42

43. Semmler A, Okulla T, Kaiser M, Seifert B, Heneka MT. Long-term neuromuscular sequelae of critical illness. J Neurol 2013; 260(1):151-7.

44. Nguyen The L, Nguyen Huu C. Critical illness polyneuropathy and myopathy in a rural area in Vietnam. J Neurol Sci 2015; 15;357(1-2):276-81.

45. Intiso D, Di Rienzo F, Fontana A, Tolfa M, Bartolo M, Copetti M. Functional outcome of critical illness polyneuropathy in patients affected by severe brain injury. Eur J Phys Rehabil Med 2017; 53(6):910-919.

46. Cunningham CJB, Finlayson HC, Henderson WR, O'Connor RJ, Travlos A. Impact of Critical Illness Polyneuromyopathy in Rehabilitation: A Prospective Observational Study. PM R 2018; 10(5):494-500.

47. Symeonidou Z, Theodoraki K, Chalkias A, Argyra E, Casale R. Critical Illness Polyneuropathy (CIP): a multicenter study on functional outcome. G Ital Med Lav Ergon 2019; 41(1):58-64.

48. Hakiki B, Draghi F, Scarpino M, Portaccio E, Romoli A, Mannini A, Atzori T, Lolli F, Macchi C, Grippo A. Critical illness polyneuromyopathy: Functional impact after severe acquired brain injuries Acta Neurol Scand 2020; 142(6):574-584.

49. Op de Coul AA, Lambregts PC, Koeman J, van Puyenbroek MJ, Ter Laak HJ, Gabreëls-Festen AA. Neuromuscular complications in patients given Pavulon (pancuronium bromide) during artificial ventilation. Clin Neurol Neurosurg 1985; 87(1):17-22

50. Gooch JL, Suchyta MR, Balbierz JM, et al. Prolonged paralysis after treatment with neuromuscular junction blocking agents. Crit Care Med 1991; 19:1125-1131.

51. Lacomis D, Giuliani MJ, Van Cott A, et al. Acute myopathy of intensive care: clinical, electromyographic, and pathological aspects. Ann Neurol $1996 ; 40: 645-654$.

52. Koch S, Spuler S, Deja M, Bierbrauer J, Dimroth A, Behse F, Spies CD, Wernecke KD, Weber-Carstens S. Critical illness myopathy is frequent: accompanying neuropathy protracts ICU discharge. J Neurol Neurosurg Psychiatry 2011; 82(3):287-93

53. Schmidt SB, Rollnik JD. Critical illness polyneuropathy (CIP) in neurological early rehabilitation: clinical and neurophysiological features. BMC Neurol 2016; 16(1):256

54. Kelmenson DA, Held N, Allen RR, Quan D, Burnham EL, Clark BJ, Ho PM, Kiser TH, Vandivier RW, Moss M. Outcomes of ICU Patients With a Discharge Diagnosis of Critical Illness Polyneuromyopathy: A Propensity-Matched Analysis. Crit Care Med 2017; 45(12):2055-2060.

55. Meyer-Frießem CH, Malewicz NM, Rath S, Ebel M, Kaisler M, Tegenthoff M, Schildhauer TA, Pogatzki-Zahn EM, Maier C, Zahn PK. Incidence, Time Course and Influence on Quality of Life of Intensive Care Unit-Acquired Weakness Symptoms in Long-Term Intensive Care Survivors. J Intensive Care Med 2020; 885066620949178. doi: 10.1177/0885066620949178.

56. Van Aerde N, Meersseman P, Debaveye Y, Wilmer A, Gunst J, Casaer MP, Bruyninckx F, Wouters PJ, Gosselink R, Van den Berghe G, Hermans G. Five-year impact of ICU-acquired neuromuscular complications: a prospective, observational study. Intensive Care Med 2020; 46(6):1184-1193.

57. De Jonghe B, Sharshar T, Hopkinson N, Outin H. Paresis following mechanical ventilation. Curr Opin Crit Care 2004; $10(1): 47-52$.

58. Garnacho-Montero J, Amaya-Villar R, García-Garmendía JL, Madrazo-Osuna J, Ortiz-Leyba C. Effect of critical illness polyneuropathy on the withdrawal from mechanical ventilation and the length of stay in septic patients. Crit Care Med, 2005;(2):349-54

59. Liu M, Luo J, Zhou J, Zhu, X. Intervention effect of neuromuscular electrical stimulation on ICU acquired weakness: A meta-analysis. Int J Nurs Sci $2020 ; 7(2): 228-237$.

Page $18 / 22$ 
60. Mehrholz J, Pohl M, Kugler J, Burridge J, Mückel S, Elsner B. Physical rehabilitation for critical illness myopathy and neuropathy. Cochrane Database Syst Rev 2015; (3):CD010942.

61. Intiso D. ICU-acquired weakness: should medical sovereignty belong to any specialist? Crit Care 2018; 22(1):1 doi: 10.1186/s13054-017-1923-7.

\section{Appendix}

Appendix 1 Excluded studies that enrolled subjects with CIPNM. 


\begin{tabular}{|c|c|c|c|c|c|c|c|}
\hline Authors & $\begin{array}{l}\text { Study/ } \\
\text { Design/ } \\
\text { setting }\end{array}$ & $\begin{array}{l}\text { N/followed/ } \\
\text { CIPNM type }\end{array}$ & Etiology & Follow-up & $\begin{array}{l}\text { Functional } \\
\text { measures/ } \\
\text { other }\end{array}$ & $\begin{array}{l}\text { Other } \\
\text { measures }\end{array}$ & Outcome \\
\hline $\begin{array}{l}\text { Op de Cul } \\
\text { A et al }{ }^{48} \\
(1985)\end{array}$ & $\begin{array}{l}\text { case series; } \\
\text { ICU }\end{array}$ & $\begin{array}{l}\mathrm{N}=12 / 9 ; \\
\mathrm{CIP}=4 ; \\
\mathrm{CIP} / \mathrm{CIM}=3\end{array}$ & $\begin{array}{l}\text { eight pts } \\
\text { had } \\
\text { multiple } \\
\text { trauma. Of } \\
\text { these, } 5 \text { pts } \\
\text { with brain } \\
\text { concussion }\end{array}$ & $\begin{array}{l}5 \text { wks- } 5 \\
\text { mos }\end{array}$ & none & $\begin{array}{l}\text { EMG, } \\
\text { histological } \\
\text { examination }\end{array}$ & $\begin{array}{l}7(58 \%) \text { pts gained complete } \\
\text { functional recovery, } 2(16.6 \%) \text { had } \\
\text { incomplete recovery and } 3 \text { pts died }\end{array}$ \\
\hline $\begin{array}{l}\text { Gooch JL } \\
\text { et al }{ }^{49} \\
(1991)\end{array}$ & $\begin{array}{l}\text { case series; } \\
\text { retrospective }\end{array}$ & $\begin{array}{l}\mathrm{N}=12 ; \\
\text { (mean age } \\
34.2 \pm 20.5 \\
\mathrm{yrs} \text { ) }\end{array}$ & $\begin{array}{l}\text { medical } \\
\text { and } \\
\text { pulmonary } \\
\text { disorders }\end{array}$ & $3-6$ mos & none & $\begin{array}{l}\text { EMG, muscle } \\
\text { biopsy }(2 \\
\text { pts) }\end{array}$ & $\begin{array}{l}8(66.6 \%) \text { pts had complete functional } \\
\text { recovery within } 6 \text { months; } 2(16.6 \%) \\
\text { pts incomplete functional recovery } \\
\text { and } 2 \text { pts pts died }(17 \%)\end{array}$ \\
\hline $\begin{array}{l}\text { Lacomis D } \\
\text { et al }{ }^{50} \\
(1996)\end{array}$ & $\begin{array}{l}\text { case series; } \\
\text { retrospective; } \\
\text { ICU }\end{array}$ & $\begin{array}{l}N=14 / 10 ; \\
9 \text { M, } 5 \text { F; } \\
\text { CIM = } 10\end{array}$ & $\begin{array}{l}\text { medical } \\
\text { disorders } \\
\text { and organ } \\
\text { transplant, } \\
\text { in } \\
\text { particular } \\
\text { liver } \\
\text { transplant }\end{array}$ & $\begin{array}{l}1 \mathrm{wk}-12 \\
\text { mos }\end{array}$ & none & $\begin{array}{l}\text { clinical, } \\
\text { EMG/ENG, } \\
\text { histologic } \\
\text { examination } \\
\text { (14 pts) }\end{array}$ & $\begin{array}{l}5 \text { pts complete functional recovery; } \\
5 \text { pts incomplete functional recovery: } \\
3 \text { pts were walking with assistance at } \\
6 \text { weeks, } 3 \text { and } 4 \text { months, respectively; } \\
1 \text { pt had moderate weakness at } 2 \\
\text { months, } 1 \text { pt was ventilator-dependent } \\
\text { at } 2 \text { months; } \\
2 \text { pts died }\end{array}$ \\
\hline $\begin{array}{l}\text { Koch S et } \\
\mathrm{al}^{51}(2011)\end{array}$ & $\begin{array}{l}\text { single center; } \\
\text { cohort; } \\
\text { prospective } \\
\text { observational; } \\
\text { ICU }\end{array}$ & $\begin{array}{l}N=53 ; \\
\mathrm{CIP}=1 ; \\
\mathrm{CIM}=16 ; \\
\mathrm{CIP} / \mathrm{CIM}=20 ; \\
\text { Unspecified= } \\
3\end{array}$ & $\begin{array}{l}\text { severe } \\
\text { head } \\
\text { trauma } \\
\text { were } \\
\text { excluded }\end{array}$ & & none & $\begin{array}{l}\text { MRC; EMG; } \\
\text { dmMCAP, } \\
\text { neCMAP }\end{array}$ & $\begin{array}{l}\text { At discharge from ICU, } 25 \% \text { of } \\
\text { patients with isolated CIM showed } \\
\text { electrophysiological signs of recovery } \\
\text { and significantly lower degrees of } \\
\text { weakness. Recovery could not be } \\
\text { observed in patients with combined } \\
\text { CIM/CIP }\end{array}$ \\
\hline $\begin{array}{l}\text { Schmidt B } \\
\text { and Rollnik } \\
D^{52}(2016)\end{array}$ & $\begin{array}{l}\text { cohort; } \\
\text { single center; } \\
\text { retrospective; } \\
\text { rehabilitation }\end{array}$ & $\begin{array}{l}\mathrm{N}=159(90 \\
\mathrm{M}, 69 \mathrm{~F}, \\
\text { mean age } \\
66 \pm 11) \\
\mathrm{CIP}=103 \\
\text { clinically } \\
\text { diagnosed; } \\
\mathrm{CIP}=56 \\
\text { diagnosed by } \\
\text { ES exam }\end{array}$ & $\begin{array}{l}\mathrm{N}=52 \mathrm{pts} \\
\text { had } \\
\text { diabetes } \\
\text { mellitus }\end{array}$ & $\begin{array}{l}\text { not } \\
\text { reported }\end{array}$ & $\begin{array}{l}\text { Barthel } \\
\text { Index; } \\
\text { (value } \\
\text { score not } \\
\text { reported) }\end{array}$ & $\begin{array}{l}\text { neurography; } \\
\text { LOS }\end{array}$ & $\begin{array}{l}\text { Outcome and prognosis of patients } \\
\text { with confirmed CIP was not different } \\
\text { from patients without neuropathy. }\end{array}$ \\
\hline $\begin{array}{l}\text { Kelmenson } \\
\text { DA et al }{ }^{53} \\
(2018)\end{array}$ & $\begin{array}{l}\text { cohort with } \\
\text { propensity } \\
\text { score } \\
\text { matched } \\
\text { analysis; } \\
\text { retrospective }\end{array}$ & $\begin{array}{l}\mathrm{N}=3.567 \text { pts } \\
\text { with a } \\
\text { discharge } \\
\text { diagnosis of } \\
\text { CIPNM by } \\
\text { ICD-9 code } \\
\text { during } 2010- \\
2014\end{array}$ & $\begin{array}{l}\text { medical } \\
\text { and } \\
\text { pulmonary } \\
\text { disorders }\end{array}$ & $\begin{array}{l}\text { not } \\
\text { reported }\end{array}$ & $\begin{array}{l}\text { not } \\
\text { reported }\end{array}$ & & $\begin{array}{l}\text { Patients with a discharge diagnosis } \\
\text { of CIPNM had fewer } 28 \text {-day hospital } \\
\text { free days (6 [0.1] vs } 7.4 \text { [0.1] days, } \\
\mathrm{p}<0.0001) \text {, fewer } 28 \text {-day ventilator } \\
\text { free days }(15.7[0.2] \text { vs } 17.5 \text { [0.2] days, } \\
\text { p<0.0001), were less likely to be } \\
\text { discharged home }(15.3 \% \text { vs } 32.8 \% \text {, } \\
p<0.0001)\end{array}$ \\
\hline
\end{tabular}




\begin{tabular}{|c|c|c|c|c|c|c|c|}
\hline Authors & $\begin{array}{l}\text { Study/ } \\
\text { Design/ } \\
\text { setting }\end{array}$ & $\begin{array}{l}\text { N/followed/ } \\
\text { CIPNM type }\end{array}$ & Etiology & Follow-up & $\begin{array}{l}\text { Functional } \\
\text { measures/ } \\
\text { other }\end{array}$ & $\begin{array}{l}\text { Other } \\
\text { measures }\end{array}$ & Outcome \\
\hline $\begin{array}{l}\text { Meyer- } \\
\text { Frießem } \\
\mathrm{CH} \text { et al }{ }^{54} \\
(2020)\end{array}$ & $\begin{array}{l}\text { cohort study; } \\
\text { single center }\end{array}$ & $\begin{array}{l}\mathrm{N}=149 \text { ICU } \\
\text { survivors; } \\
\text { (mean age } \\
59.5 \pm 13.4 \\
\text { yrs) } \\
\mathrm{N}=95 \text { pts } \\
\text { with } \\
\text { ICUAW } \\
\text { diagnosis } \\
\text { according the } \\
\text { German } \\
\text { International } \\
\text { Classification } \\
\text { of Diseases- } \\
10 \text { (ICD-10) } \\
2007-2017 ; \\
\mathrm{N}=33 \text { pts } \\
\text { without } \\
\text { ICUAW }\end{array}$ & $\begin{array}{l}\text { heart } \\
\text { disease } \\
\text { and } \\
\text { trauma or } \\
\text { burn } \\
\text { injuries }\end{array}$ & $\begin{array}{l}\text { ICU } \\
\text { discharge- } \\
10 \text { yrs }\end{array}$ & $\begin{array}{l}\text { not } \\
\text { reported }\end{array}$ & $\begin{array}{l}\text { phone } \\
\text { interview }\end{array}$ & $\begin{array}{l}\text { Of all surveyed ICU survivors, } 40 \% \\
\text { reported persistent symptoms at the } \\
\text { time of the interview. } 62 \% \text { had } \\
\text { persistent symptoms up to } 10 y \text { after } \\
\text { ICU (5-10y: } 46 \% \text { ). Only } 37 \% \text { of } \\
\text { participants reported a complete } \\
\text { recovery of symptoms, significantly } \\
\text { associated with an initially low } \\
\text { number of symptoms after ICU ( } p< \\
0.0001) .73 .7 \% \text { of individuals reported } \\
\text { impaired QoL, with } 29.8 \% \text { describing } \\
\text { the impairment as "severe" and } 65 \% \\
(n=37 / 57) \text { complaining about } \\
\text { limitations in terms of daily life and } \\
\text { leisure time }\end{array}$ \\
\hline
\end{tabular}

Legend: dmCMAP= direct muscle stimulation; ES= electrophysiological studies; ICD-9= International Classification of Diseases -9 ; ICU= Intensive Care Unit; MRC = Medical Research Council scale; LOS= length of stay; neCMAP= nerve stimulation.

\section{Figures}



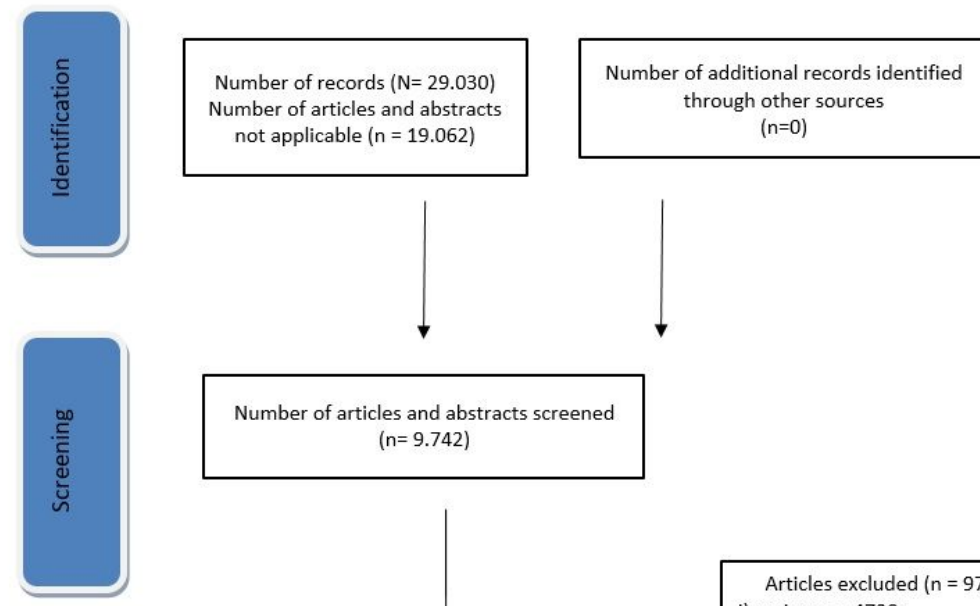

Number of articles and abstracts screened

$(n=9.742)$

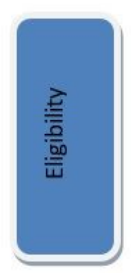

Number of full test papers assessed for

eligibility
$(n=36)$

eligibility
$(n=36)$

Articles excluded $(n=9707)$ i) review $n=4728$; ii) children $n=4910$; iii) ICUAW $\mathrm{n}=58$ (review) iV) Covid $-19=11$
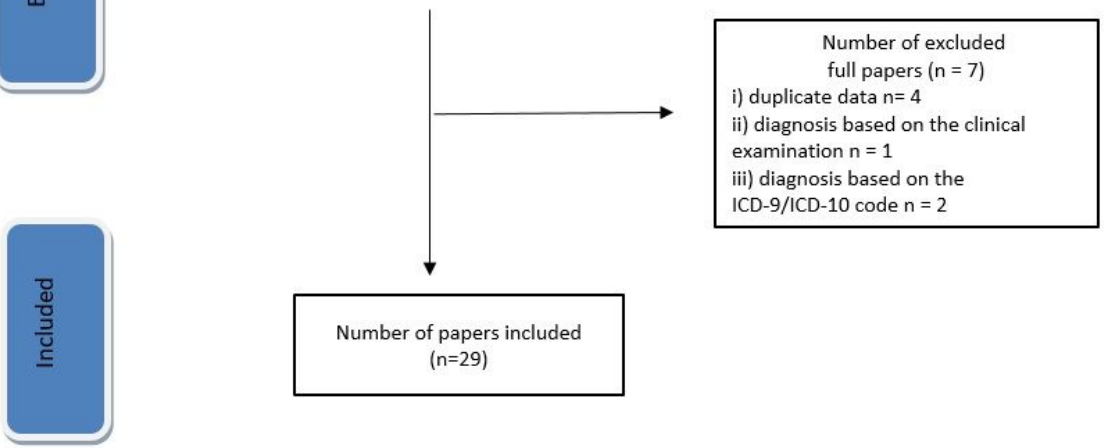

Figure 1

Preferred Reporting Items for Systematic Reviews and Meta-Analyses (PRISMA) diagram depicting the selection of articles for the study. 